\title{
Master Schedule for CY-1981 \\ Hanford Environmental \\ Surveillance Routine Program
}

\author{
P. J. Blumer \\ M. J. Sula \\ P. A. Eddy
}

December 1980

Prepared for the U.S. Department of Energy under Contract DE-AC06-76RLO 1830

Pacific Northwest Laboratory

Operated for the U.S. Department of Energy

by Battelle Memorial Institute 


\title{
NOTICE
}

This report was prepared as an account of work sponsored by the United States Government. Neither the United States nor the Department of Energy, nor any of their employees. nor any of their contractors, subcontractors. or their employees, makes any warranty, express or implied, or assumes any legal liability or responsibility for the accuracy, completeness or usefulness of any information, apparatus, product or process disclosed, or represents that its use would not infringe privately owned rights.

The views, opinions and conclusions contained in this report are those of the contractor and do not necessarily represent those of the United States Government or the United States Department of Energy.

\author{
PACIFIC NORTHWEST LABORATORY \\ operated by \\ BATTELLE \\ for the \\ UNITED STATES DEPARTMENT OF ENERGY \\ Under Contract DE-AC06-76RLO 1830
}
Printed in the United States of America
Available irom
National Technical information Service
United States Department of Commerce
5285 Port Royal Road
Springtield, Virginia 22151

Price: Printed Copy 5 $\therefore$ Microfiche $\$ 3.00$

NTIS

-Pages Selling Price

\begin{tabular}{|c|c|}
\hline $001-025$ & $\$ 4.00$ \\
\hline $026-050$ & $\$ 4.50$ \\
\hline $051-075$ & $\$ 5.25$ \\
\hline $076-100$ & $\$ 6.00$ \\
\hline $101-125$ & $\$ 6.50$ \\
\hline $126-150$ & $\$ 7.25$ \\
\hline $151-175$ & $\$ 8.00$ \\
\hline $176 \cdot 200$ & $\$ 9.00$ \\
\hline $1-225$ & $\$ 9.25$ \\
\hline $6-250$ & 59.50 \\
\hline 1.275 & $\$ 10.75$ \\
\hline $6-300$ & $\$ 11.00$ \\
\hline
\end{tabular}


PNL -3639

UC-41

\title{
33679000561425
}

\author{
MASTER SCHEDULE FOR CY-1981 \\ HANFORD ENVIRONMENTAL SURVEILLANCE \\ ROUTINE PROGRAM
}

P. J. Blumer

M. J. Sula

P. A. Eddy

December 1980

Prepared for

the U. S. Department of Energy

under Contract DE-AC06-76RLO 1830

Pacific Northwest Laboratory

Richland, Washington 99352 
, 


\section{CONTENTS}

\begin{tabular}{|c|c|c|c|c|c|c|c|c|c|c|c|c|c|}
\hline INTRODUCT & ION & . & . & . & . & • & - & - & - & . & • & • & 1 \\
\hline SCHEDULE & . & . & . & . & . & . & . & . & • & . & . & . & 3 \\
\hline I. & AIR . & . & - & - & . & - & - & - & • & . & • & . & 3 \\
\hline II. & COLUMBIA & RIVER & . & . & . & $\cdot$ & • & $\cdot$ & $\cdot$ & . & $\cdot$ & . & 9 \\
\hline II I. & SANITARY & WATER & . & - & . & • & • & $\cdot$ & • & . & • & . & 10 \\
\hline IV. & SURFACE $h$ & WATER & . & - & . & • & • & $\cdot$ & . & . & • & . & 11 \\
\hline v. & GROUND WA & IATER & . & - & . & - & - & $\cdot$ & • & . & • & . & 12 \\
\hline VI. & FOODSTUFF & & . & . & . & • & • & • & • & . & • & . & 24 \\
\hline VII. & WILDLIFE & . & . & - & . & • & • & $\cdot$ & • & . & • & $\cdot$ & 26 \\
\hline VIII. & SOIL AND & VEGET & ATION & . & . & - & . & • & • & . & • & . & 28 \\
\hline IX. & EXTERNAL & RADIA & TION & MEA & REMENT & & - & • & • & . & • & & 30 \\
\hline$x$. & PORTABLE & INSTR & UMENT & SU & EYS & . & - & $\cdot$ & $\bullet$ & . & . & & 33 \\
\hline XI. & SURVE ILLA & ANCE 0 & $F$ WAS & & SPOSAL & & & • & • & . & • & 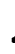 & 35 \\
\hline
\end{tabular}




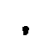




\section{INTRODUCTION}

This report provides the current schedule of data collection for the routine environmental surveillance program at the Hanford Site. The program is sponsored by the Department of Energy and is conducted by the Environmental Evaluations Section of Pacific Northwest Laboratory. (a) Questions about specific entries should be referred to the authors since modifications to the schedule are made during the year and special areas of study, usually of short duration, are not scheduled.

The environmental surveillance program objectives are to evaluate the levels of radioactive and nonradioactive pollutants in the Hanford environs, as required in Manual Chapter 0513, and to monitor Hanford operations for compliance with applicable environmental criteria given in Manual Chapter 0524 and Washington State Water Quality Standards. Air quality data are obtained in a separate program administered by the Hanford Environmental Health Foundation. The collection schedule for potable water is shown but it is not part of the routine environmental surveillance program. Water quality data for Hanford Site potable water systems are published each year by the Hanford Environmental Health Foundation.

The data collected are available in routine reports issued by the Environmental Evaluations staff. Groundwater data and evaluations are reported in the series, "Radiological Status of the Groundwater Beneath the Hanford Project. The latest issue is PNL 3346 for $\mathrm{CY}-1979$. Data from locations within the plant boundaries are presented in the annual report series, "Environmental Status of the Hanford Site." The most recent report in this series is PNL-3284 for CY-1979. Data from offsite locations are presented annually in the "Environmental Surveillance at Hanford" series. The latest report in this series is PNL-3283 for CY-1979.

(a) Operated by Battelle Memorial Institute. 


\section{Frequency Symbols Used}

$$
\begin{aligned}
D & - \text { Daily } \\
W & - \text { Weekly } \\
B W & - \text { Biweekly (every } 2 \text { wk) } \\
B & - \text { Monthly } \\
B M & - \text { Bimonthly (every } 2 \mathrm{mo})
\end{aligned}
$$

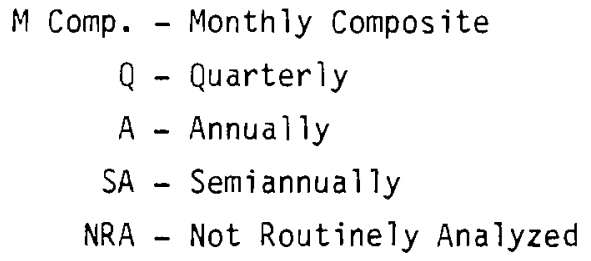


I. Air

\begin{tabular}{|c|c|c|c|c|}
\hline \multirow{2}{*}{ Location } & \multicolumn{4}{|c|}{ Filter } \\
\hline & EMA\# & Frequency & Analyses & $\begin{array}{l}\text { Composite(d) } \\
\text { Group } \\
\end{array}$ \\
\hline \multicolumn{5}{|l|}{ Onsite } \\
\hline $100-K$ & 0058 & BW & Beta & 100 Areas \\
\hline $100-N$ (WPPSS) & 1529 & BW & Beta & 100 Areas \\
\hline $100-D$ & 1074 & BW & Beta & 100 Areas \\
\hline 100-A Fire Station & 6154 & BW & Beta & 100 Areas \\
\hline 200 WEC & 0046 & BW & Beta, Alpha & 200 west \\
\hline 200 WNE & 1370 & BW & Beta & 200 West \\
\hline 200 WWC & 0045 & BW & Beta & 200 west \\
\hline Redox & 0028 & BW & Beta, Alpha & 200 West \\
\hline 200 ESE & 0043 & $\mathrm{BW}$ & Beta, Alpha & 200 East \\
\hline 200 ENC & 1401 & $\mathrm{BW}$ & Beta, Alpha & 200 East \\
\hline $200 \mathrm{EEC}$ & 0042 & $\mathrm{BW}$ & Beta, Alpha & 200 East \\
\hline 200 EWC & 0041 & BW & Beta & 200 East \\
\hline Hanford & 0057 & $B W$ & Beta & IE \\
\hline Wye Barricade & 0924 & $\mathrm{BW}$ & Beta, Alpha & IE \\
\hline $400 \mathrm{E}$ & 6308 & BW & Beta, Alpha & 400 Area \\
\hline $400 \mathrm{~W}$ & 6455 & BW & Beta, Alpha & 400 Area \\
\hline $400 \mathrm{~S}$ & 6456 & $\mathrm{BW}$ & Beta, Alpha & 400 Area \\
\hline $400 \mathrm{~N}$ & 6457 & $\mathrm{BW}$ & Beta, Alpha & 400 Area \\
\hline 3705 Building & 1531 & BW & Beta & 300 Area \\
\hline ACRMS & 1793 & $\mathrm{BW}$ & Beta & 300 Area \\
\hline 300 SW Gate & 6148 & BW & Beta & 300 Area \\
\hline 300 South Gate & 6150 & BW & Beta, Alpha & 300 Area \\
\hline 300 Pond & 1543 & BW & Beta & 300 Area \\
\hline RRC CP $\$ 64$ & 6182 & BW & Beta, Alpha & ISE \\
\hline 1100 Area & 6396 & $\mathrm{BW}$ & Beta & ISE \\
\hline Prosser Barricade & 0055 & BW & Beta, Alpha & ISW \\
\hline ERC & 0929 & $\mathrm{BW}$ & Beta & ISW \\
\hline Rattlesnake Springs & 0972 & $B W$ & Beta & ISW \\
\hline Yakima Barricade & 1650 & $\mathrm{BW}$ & Beta & INW \\
\hline Vernita Bridge & 1651 & BW & Beta & INW \\
\hline
\end{tabular}

(a) Composite group for filter analyses shown on page 7 . 
I. Air (conta)

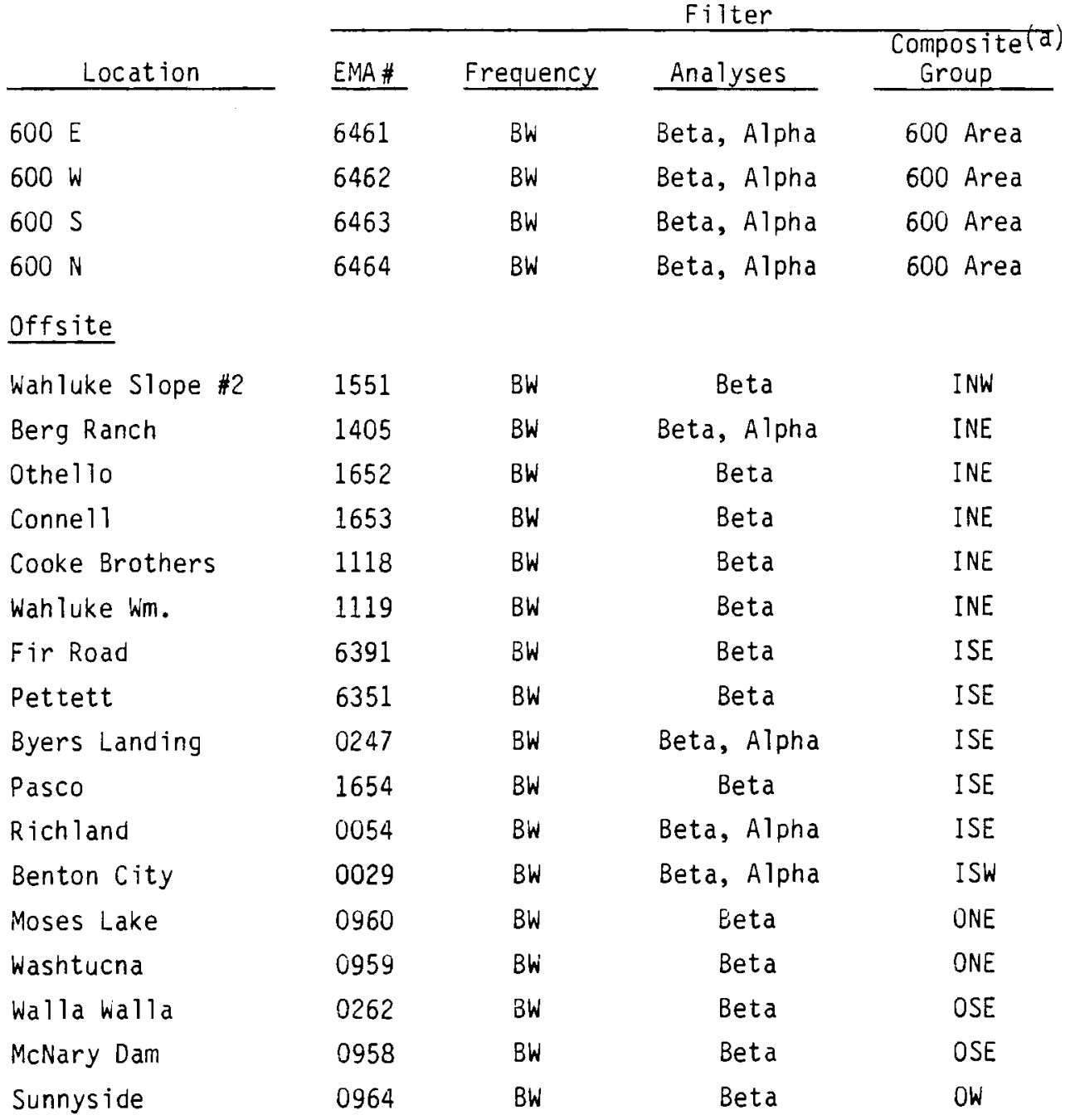

(a) Composite group for filter analyses shown on page 7 . 
I. Air (conto)

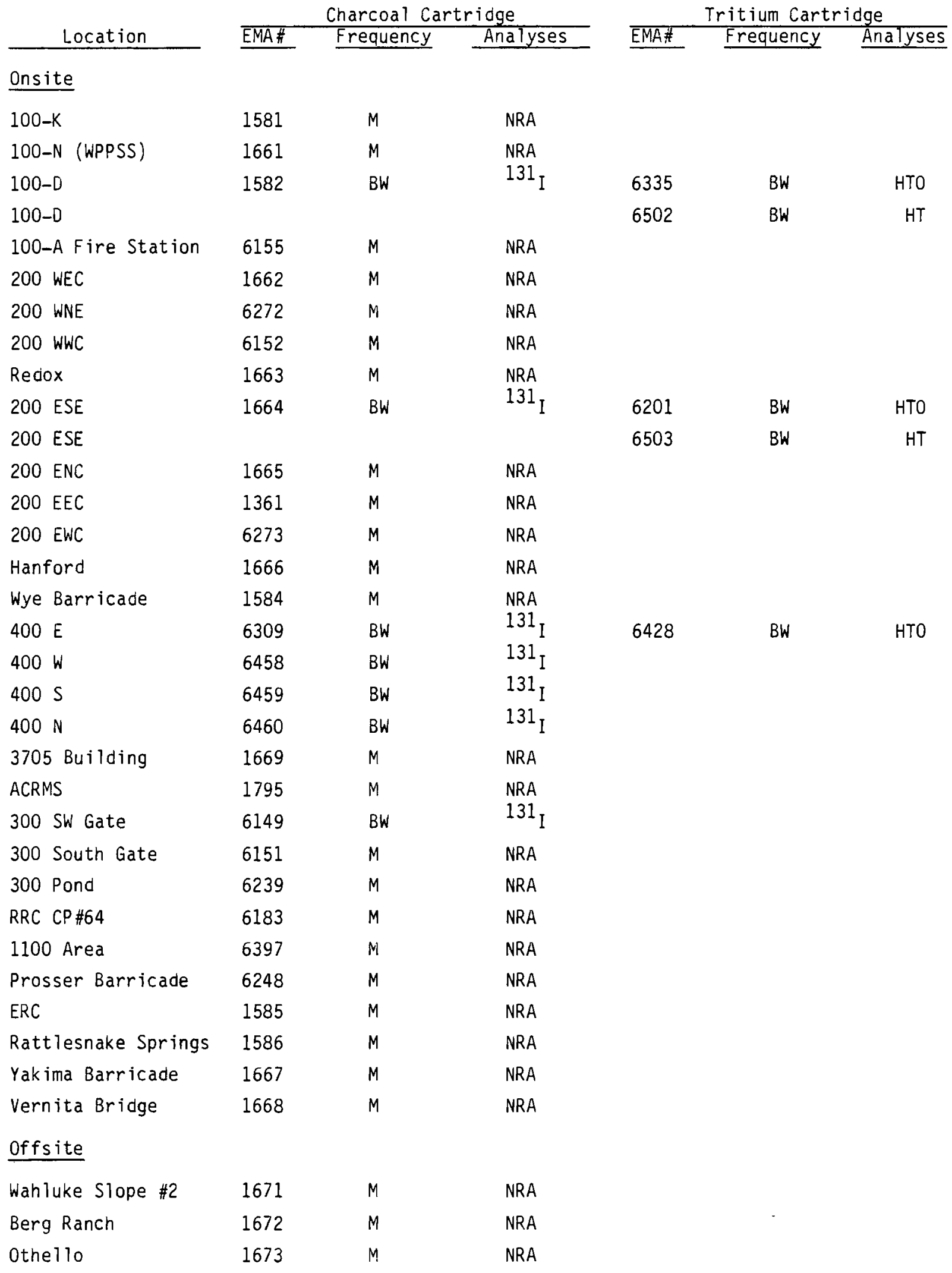


I. Air (contd)

\begin{tabular}{|c|c|c|c|c|c|c|}
\hline \multirow{2}{*}{ Location } & \multicolumn{3}{|c|}{ Charcoal Cartriage } & \multicolumn{3}{|c|}{ Tritium Cartridge } \\
\hline & EMA\# & Frequency & Analyses & EMA\# & Frequency & Analyses \\
\hline Connell & 1674 & M & NRA & & & \\
\hline Cooke Brothers & 1675 & $M$ & NRA & & & \\
\hline Wahluke wm. & 1676 & M & NRA & & & \\
\hline Fir Road & 6392 & BW & ${ }^{131} \mathrm{I}$ & 6393 & BW & HTO \\
\hline Fir Road & & & & 6513 & $B W$ & HT \\
\hline Pettett & 6352 & $B W$ & $131_{I}$ & & & \\
\hline Byers Landing & 0246 & $\mathrm{BW}$ & ${ }^{131}$ I & & & \\
\hline Pasco & 1678 & M & NRA & & & \\
\hline Richland & 0231 & $\mathrm{BW}$ & ${ }^{131} 1_{I}$ & 6207 & $\mathrm{BW}$ & HTO \\
\hline Richland & & & & 6514 & $\mathrm{BW}$ & HT \\
\hline Benton City & 1670 & BW & ${ }^{131} 1_{I}$ & 6411 & $B W$ & HTO \\
\hline Benton City & & & & 6515 & $B W$ & HT \\
\hline Moses Lake & 1682 & M & NRA & & & \\
\hline Washtucna & 1683 & M & NRA & & & \\
\hline Walla Walla & 0261 & M & NRA & & & \\
\hline McNary Dam & 1684 & M & NRA & & & \\
\hline Sunnyside & 1680 & $B W$ & ${ }^{131} \mathrm{I}$ & & & \\
\hline
\end{tabular}


I. Air (contd)

\begin{tabular}{|c|c|c|c|}
\hline Composite Group & EMA\# & Frequency & Analyses \\
\hline $\begin{array}{l}200 \text { East } \\
200 \text { ENC } \\
200 \text { EWC } \\
200 \text { ESE } \\
200 \text { EEC }\end{array}$ & 1749 & $\begin{array}{l}M \\
Q\end{array}$ & $\begin{array}{l}\text { Gamma scan } \\
{ }^{90} \mathrm{Sr} \text {, Pu-total }\end{array}$ \\
\hline $\begin{array}{l}200 \text { West } \\
200 \text { WEC } \\
200 \text { WWC } \\
\text { Redox } \\
200 \text { WNE }\end{array}$ & 1751 & $\begin{array}{l}M \\
Q\end{array}$ & $\begin{array}{l}\text { Gamma scan } \\
90_{\mathrm{Sr}} \text {, Pu-total }\end{array}$ \\
\hline $\begin{array}{l}300 \text { Area } \\
300 \text { Area, } 3705 \text { Building } \\
\text { ACRMS } \\
300 \text { South Gate } \\
300 \text { Southwest Gate } \\
300 \text { Pond }\end{array}$ & 1752 & $\begin{array}{l}M \\
Q\end{array}$ & $\begin{array}{l}\text { Gamma scan } \\
90 \mathrm{Sr} \text {, Pu-total }\end{array}$ \\
\hline $\begin{array}{l}400 \text { Area } \\
400 \mathrm{E} \\
400 \mathrm{~W} \\
400 \mathrm{~S} \\
400 \mathrm{~N}\end{array}$ & 6465 & $\begin{array}{l}M \\
Q\end{array}$ & $\begin{array}{l}\text { Gamma scan } \\
{ }^{90} \mathrm{Sr} \text {, Pu-total }\end{array}$ \\
\hline $\begin{array}{l}100 \text { Areas } \\
100 \text { Area Fire Station } \\
100-K \\
100-N \\
100-D\end{array}$ & 1753 & $\begin{array}{l}M \\
Q\end{array}$ & $\begin{array}{l}\text { Gamma scan } \\
{ }^{90} \mathrm{Sr} \text {, Pu-total }\end{array}$ \\
\hline $\begin{array}{l}600 \text { Area } \\
600 \mathrm{E} \\
600 \mathrm{~W} \\
600 \mathrm{~S} \\
600 \mathrm{~N}\end{array}$ & 6466 & $\begin{array}{l}M \\
Q\end{array}$ & $\begin{array}{l}\text { Gamma scan } \\
{ }^{90} \text { Sr, Pu-tota } 1\end{array}$ \\
\hline $\begin{array}{l}\text { Inner Eastern Sector } \\
\text { Hanford }\end{array}$ & 1754 & $\begin{array}{l}M \\
Q\end{array}$ & $\begin{array}{l}\text { Garma scan } \\
90_{S r} \text {, Pu-total }\end{array}$ \\
\hline
\end{tabular}


I. Air (contd)

\begin{tabular}{|c|c|c|c|}
\hline Composite Group & EMA\# & Frequency & Analyses \\
\hline $\begin{array}{l}\text { Inner Southeast Sector } \\
\text { RRC CP\#64 } \\
1100 \text { Area } \\
\text { Richland } \\
\text { Pasco } \\
\text { Byers Landing } \\
\text { Fir Road } \\
\text { Pettett }\end{array}$ & 1745 & $\begin{array}{l}M \\
Q\end{array}$ & $\begin{array}{l}\text { Gamma scan } \\
{ }^{90} \mathrm{Sr}, \text { Pu-total }\end{array}$ \\
\hline $\begin{array}{l}\text { Inner Southwest Sector } \\
\text { Rattlesnake Springs } \\
\text { ERC } \\
\text { Benton City } \\
\text { Prosser Barricade }\end{array}$ & 1746 & $M$ & $\begin{array}{l}\text { Gamma scan } \\
{ }^{90} \mathrm{Sr}, \text { Pu-total }\end{array}$ \\
\hline $\begin{array}{l}\text { Inner Northwest Sector } \\
\text { Yakima Barricade } \\
\text { Vernita } \\
\text { Wahluke \#2 }\end{array}$ & 1747 & $\begin{array}{l}M \\
Q\end{array}$ & $\begin{array}{l}\text { Gamma scan } \\
{ }^{90} \text { Sr, Pu-total }\end{array}$ \\
\hline $\begin{array}{l}\text { Inner Northeast Sector } \\
\text { Othe } 110 \\
\text { Conne } 11 \\
\text { Berg Ranch } \\
\text { Wanluke Watermaster } \\
\text { Cooke Brothers }\end{array}$ & 1743 & $\begin{array}{l}M \\
Q\end{array}$ & $\begin{array}{l}\text { Gamma scan } \\
{ }^{90} \mathrm{Sr} \text {, Pu-total }\end{array}$ \\
\hline $\begin{array}{l}\text { Outer Northeast Sector } \\
\text { Moses Lake } \\
\text { Washtucna }\end{array}$ & 1742 & $\begin{array}{l}M \\
Q\end{array}$ & $\begin{array}{l}\text { Gamma scan } \\
90 \mathrm{Sr}, \text { Pu-total }\end{array}$ \\
\hline $\begin{array}{l}\text { Outer Southeast Sector } \\
\text { Walla Walla } \\
\text { McNary }\end{array}$ & 1744 & $\begin{array}{l}M \\
Q\end{array}$ & $\begin{array}{l}\text { Gamma scan } \\
90 \mathrm{Sr} \text {, Pu-total }\end{array}$ \\
\hline $\begin{array}{l}\text { Outer Western Sector } \\
\text { Sunnyside }\end{array}$ & 1748 & $\begin{array}{l}M \\
Q\end{array}$ & $\begin{array}{l}\text { Gamma scan } \\
90 \mathrm{Sr} \text {, Pu-total }\end{array}$ \\
\hline
\end{tabular}


II. COLUMBIA RIVER

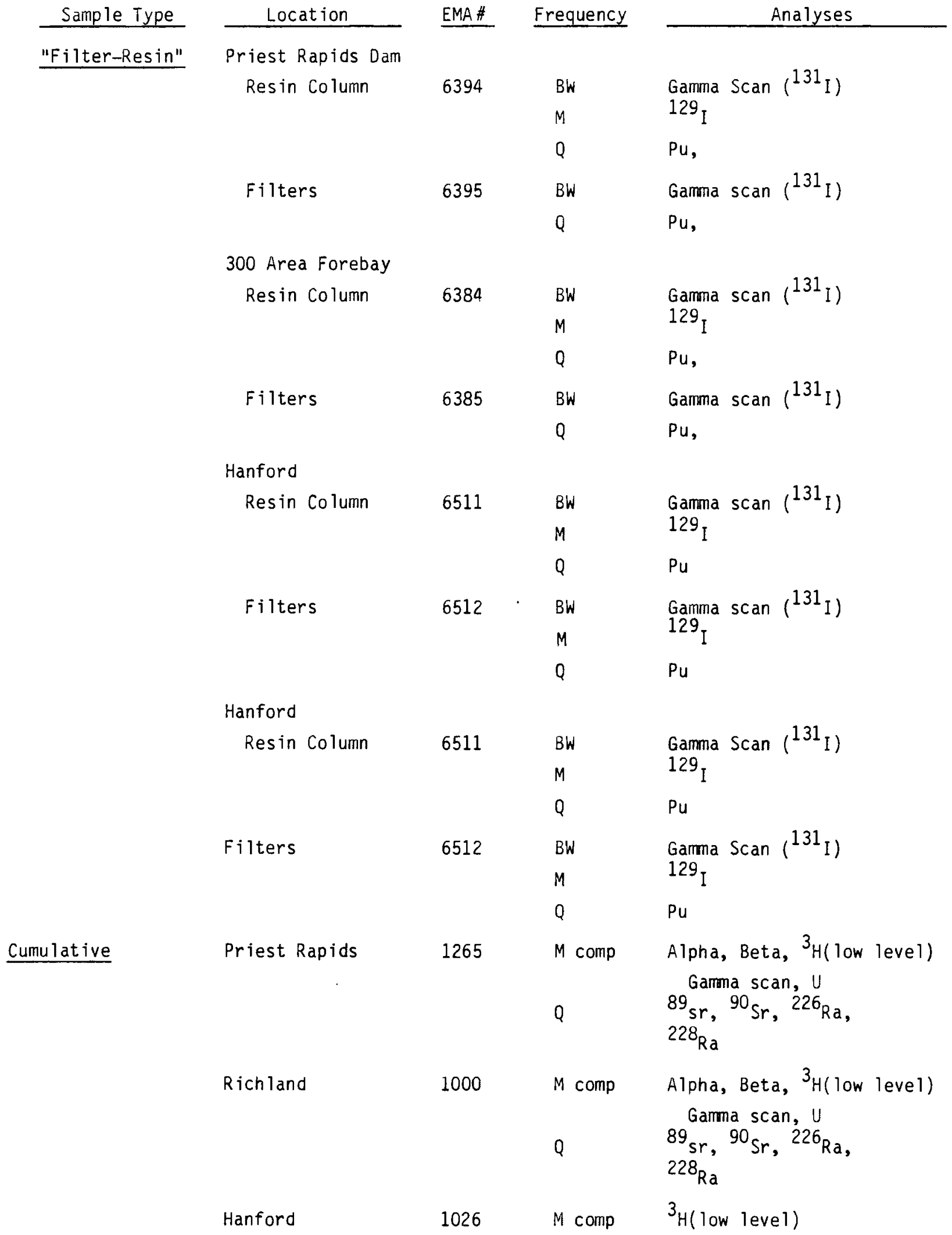


II. COLUMBIA RIVER (contd)

\begin{tabular}{|c|c|c|c|c|}
\hline Sample Type & Location & EMA\# & Frequency & Analyses \\
\hline \multirow[t]{4}{*}{ Grab } & Vernita & $\begin{array}{l}1616 \\
1204 \\
1373\end{array}$ & $\begin{array}{l}W \\
W \\
M^{*}\end{array}$ & $\begin{array}{l}\mathrm{NO}_{3} \text {, } \\
\mathrm{pH}, \text { Diss } \mathrm{O}_{2} \text {, Turbidity } \\
\text { Coliforms, Fecal coliforms, } \\
\quad \mathrm{BOD}\end{array}$ \\
\hline & Richland Forebay & 1617 & w & \\
\hline & & 1365 & $M^{\star}$ & $\begin{array}{l}\text { Coliforms, BOD, Fecal } \\
\text { coliforms }\end{array}$ \\
\hline & & 6429 & W & $\mathrm{pH}$, Diss $0_{2}$, Turbidity \\
\hline
\end{tabular}

* Performed by HEHF. 
III. SAMITARY WATER

\begin{tabular}{|c|c|c|c|c|}
\hline Sample Type & Location & EMA\# & Frequency & Analyses \\
\hline \multirow[t]{7}{*}{ Cumulative } & Richland & 1002 & W & Alpha, Beta \\
\hline & & & M comp & $\begin{array}{l}\text { Gamma scan, }{ }^{90} \mathrm{Sr},{ }^{226} 6_{\mathrm{Ra}} \text {, } \\
228_{\mathrm{Ra}}\end{array}$ \\
\hline & & 1366 & $w^{\star}$ & $\mathrm{NO}_{\overline{3}}$ \\
\hline & 300 Area & 1686 & $Q$ & $\begin{array}{l}\text { Alpha, Beta, }{ }^{90} \mathrm{Sr},{ }^{3} \mathrm{H}, \mathrm{U}, \\
226_{\mathrm{Ra}},{ }^{228_{\mathrm{Ra}}}\end{array}$ \\
\hline & & 1724 & $w^{\star}$ & $\mathrm{NO}_{\overline{3}}$ \\
\hline & FFTF & 6382 & M comp & $\begin{array}{l}\text { Alpha, Beta, }{ }^{3} \mathrm{H},{ }^{90} \mathrm{Sr} \text {, } \\
226_{\mathrm{Ra}},{ }^{228_{\mathrm{Ra}}}\end{array}$ \\
\hline & & & $Q$ & Gamma $\operatorname{scan}\left({ }^{22} \mathrm{Na}\right),{ }^{106} \mathrm{Ru}$ \\
\hline \multirow[t]{10}{*}{ Grab } & Observatory & 6381 & Q & $\begin{array}{l}\text { A lpha, Beta, }{ }^{3} \mathrm{H},{ }^{90} \mathrm{Sr}, \\
226_{\mathrm{Ra}},{ }^{228_{\mathrm{Ra}}}\end{array}$ \\
\hline & $100-0$ & 1219 & $Q$ & Alpha, Beta \\
\hline & 200 East & 6374 & $Q$ & Alpha, Beta \\
\hline & 200 West & 6375 & $Q$ & Alpha, Beta \\
\hline & 100 Area Fire & & & \\
\hline & Station & 6376 & $Q$ & Alpha, Beta \\
\hline & 200 Area Fire & & & \\
\hline & Station & 6377 & $Q$ & Alpha, Beta \\
\hline & 251 Building & 6378 & $Q$ & Alpha, Beta \\
\hline & 622-R Building & 6380 & $Q$ & Alpha, Beta \\
\hline
\end{tabular}

* Performed by HEHF. 
IV. SURFACE WATER

\begin{tabular}{|c|c|c|c|}
\hline Location & EMA\# & Frequency & Analyses \\
\hline Gable Mounta in Pond & 1054 & $\mathrm{Q}$ & Alpha, Beta, Gamma scan, ${ }^{90} \mathrm{Sr}$ \\
\hline West Lake & 6133 & $Q$ & Alpha, Beta, Gamria scan, \\
\hline B Pona & 0015 & M & Alpha, Beta, Gamma scan, ${ }^{90} \mathrm{Sr}$ \\
\hline FFTF & 6467 & $Q$ & Aipha, Beta, Gamma scan $\left({ }^{22} \mathrm{Na}\right)$ \\
\hline
\end{tabular}


V. GROUND WATER

\begin{tabular}{|c|c|c|c|}
\hline Well Number & EMA\# & Frequency & Analyses (a) \\
\hline & 600 & ea Wells & \\
\hline$(699-) 50-7$ & $\star 4817$ & $Q$ & ${ }^{3}, \mathrm{NO}_{3}, \quad \mathrm{~B}, \gamma$ \\
\hline SO-8 & $\star 4818$ & Q & ${ }^{3} \mathrm{H}, \mathrm{NO}_{3}, \beta, \gamma$ \\
\hline$S 1-7 B$ & $\star 4819$ & $Q$ & ${ }^{3} \mathrm{H}, \mathrm{NO}_{3}, \beta, \gamma$ \\
\hline$S 3-25$ & 4787 & $Q$ & ${ }^{3} \mathrm{H}, \mathrm{NO}_{3}$ \\
\hline & & A & WQ \\
\hline S3-E12 & 4553 & $Q$ & ${ }^{3} \mathrm{H}, \mathrm{NO}_{3}$ \\
\hline & & A & WQ \\
\hline S6-E4 B & 4502 & Q & $\mathrm{H}_{\mathrm{H}, \mathrm{NO}_{3}, \mathrm{U}}$ \\
\hline S6-E4 D & 4504 & $Q$ & ${ }^{3} \mathrm{H}, \mathrm{NO}_{3}, \mathrm{U}$ \\
\hline S6-E14 & 4580 & Q & $\mathrm{S}_{\mathrm{H}, \mathrm{NO}_{3}}$ \\
\hline $57-34$ & 4427 & $Q$ & ${ }^{3} \mathrm{H}, \mathrm{NO}_{3}$ \\
\hline $58-19$ & 4421 & Q & ${ }_{3}^{3}, \mathrm{NO}_{3}$ \\
\hline S11-E12A 0 & 4552 & Q & ${ }_{3}^{3} \mathrm{H}, \mathrm{NO}_{3}$ \\
\hline S11-E12A P & 4747 & Q & ${ }_{3}^{3} \mathrm{H}, \mathrm{NO}_{3}$ \\
\hline$S 12-3$ & 4424 & $Q$ & ${ }^{3} \mathrm{H}, \mathrm{NO}_{3}$ \\
\hline$S 12-29$ & 4592 & $Q$ & $\mathrm{NO}_{3}$ \\
\hline $\mathrm{S} 14-20 \mathrm{~A}$ & 4535 & Q & $\mathrm{NO}_{3}$ \\
\hline S18-51 & 4852 & Q & $\mathrm{NO}_{3}, \mathrm{~F}$ \\
\hline S19-11 & 4780 & SA & $\mathrm{NO}_{3}$ \\
\hline S19-E13 & 4802 & $Q$ & $\mathrm{NO}_{3}, \mathrm{~F}, \mathrm{U}$ \\
\hline & & A & WQ \\
\hline S24-19 & 4510 & SA & $\mathrm{NO}_{3}$ \\
\hline S27-E14 & 4413 & M & $\mathrm{NO}_{3}, \mathrm{U}, \mathrm{F}, \mathrm{Cr}^{\top \mathrm{G}}$ \\
\hline S29-E12 & 4803 & Q & $\mathrm{NO}_{3}, \mathrm{~F}, \mathrm{U}$ \\
\hline S30-E15 A & 4804 & $Q$ & $\mathrm{NO}_{3}, \mathrm{~F}, \mathrm{U}, \mathrm{Cr}^{+6}$ \\
\hline$S 31-1 P$ & 4745 & Q & ${ }^{3} \mathrm{H}, \mathrm{NO}_{3}$ \\
\hline & & A & WQ \\
\hline $1-18$ & 4513 & $Q$ & ${ }^{3} \mathrm{H}, \mathrm{NO}_{3}$ \\
\hline $2-3$ & $\star 4423$ & M & $\mathrm{S}_{\mathrm{H}}, \mathrm{NO}_{3}$ \\
\hline & & Q & $\beta, \gamma$ \\
\hline & & A & WQ \\
\hline
\end{tabular}

(a) Water quality analyses (designated WQ) include the following: $\mathrm{pH}$, Conductance, $\mathrm{Ca}, \mathrm{Mg}, \mathrm{Na}, \mathrm{CO}_{3}, \mathrm{HCO}_{3}, \mathrm{~K}, \mathrm{~B}$, $\mathrm{NO}_{3}-\mathrm{N}, \mathrm{Cl}, \mathrm{SO}_{4}-\mathrm{S}$, and dissolved solids.

* FFTF Preoperational Survey. 
V. GROUND WATER (contd)

\begin{tabular}{|c|c|c|c|}
\hline We 11 Number & EMA\# & Frequency & Analyses (a) \\
\hline$(699-) 2-7$ & 4758 & A & WQ \\
\hline $2-33$ & 4526 & $Q$ & ${ }^{3} \mathrm{H}, \mathrm{NO}_{3}$ \\
\hline $3-45$ & 4593 & $Q$ & $\mathrm{NO}_{3}$ \\
\hline & & $S A$ & ${ }^{3} \mathrm{H}$ \\
\hline 4-E6 & 4620 & $Q$ & ${ }^{3} \mathrm{H}, \mathrm{NO}_{3}$ \\
\hline $8-17$ & 4426 & $Q$ & $\mathrm{~B},{ }^{3} \mathrm{H}, \mathrm{NO}_{3}, \gamma$ \\
\hline $8-25$ & 4788 & $Q$ & $\mathrm{~B},{ }^{3} \mathrm{H}, \mathrm{NO}_{3}, \mathrm{r}$ \\
\hline $8-32$ & 4420 & $Q$ & ${ }^{3} \mathrm{H}, \mathrm{NO}_{3}$ \\
\hline $9-E 2$ & 4519 & Q & ${ }^{3} \mathrm{H}, \mathrm{NO}_{3}$ \\
\hline 10-E12 & 4581 & $Q$ & ${ }^{3} \mathrm{H}, \mathrm{NO}_{3}$ \\
\hline $10-54$ & 4428 & A & WQ \\
\hline $13-1 \mathrm{~A}$ & 4830 & $Q$ & ${ }^{3} \mathrm{H}, \mathrm{NO}_{3}$ \\
\hline & & SA & $\gamma_{2}^{\gamma}$ \\
\hline $13-1 B$ & 4831 & $Q$ & ${ }^{3} \mathrm{H}, \mathrm{NO}_{3}$ \\
\hline & & SA & $\gamma$ \\
\hline $13-1 C$ & 4854 & $Q$ & ${ }^{3} \mathrm{H}, \mathrm{NO}_{3}$ \\
\hline & & SA & $\gamma$ \\
\hline $13-64$ & 4429 & $Q$ & ${ }^{3} \mathrm{H}, \mathrm{NO}_{3}$ \\
\hline 14-E6 P & 4700 & $Q$ & ${ }^{3} \mathrm{H}, \mathrm{NO}_{3}$ \\
\hline 14-E6 0 & 4701 & $Q$ & ${ }^{3} \mathrm{H}, \mathrm{NO}_{3}$ \\
\hline 14-E6 R & 4702 & $Q$ & ${ }^{3} \mathrm{H}, \mathrm{NO}_{3}$ \\
\hline 14-E6 S & 4703 & $Q$ & ${ }^{3} \mathrm{H}, \mathrm{NO}_{3}$ \\
\hline 14-E6 T & 4766 & $Q$ & ${ }^{3} \mathrm{H}, \mathrm{NO}_{3}$ \\
\hline $14-38$ & 4527 & $Q$ & ${ }^{3} \mathrm{H}, \mathrm{NO}_{3}$ \\
\hline $14-47$ & 4608 & $Q$ & ${ }^{3} \mathrm{H}, \mathrm{NO}_{3}$ \\
\hline & & A & WQ \\
\hline $15-15 B$ & 4810 & $Q$ & ${ }^{3}{ }^{3} \mathrm{H}, \mathrm{NO}_{3}, \gamma$ \\
\hline $15-26$ & 4464 & $Q$ & ${ }^{8}{ }^{3} \mathrm{H}, \mathrm{NO}_{3}, r$ \\
\hline & & A & \\
\hline $17-5$ & 4422 & $Q$ & ${ }_{8}^{3} \mathrm{H}, \mathrm{NO}_{3}, \mathrm{r}$ \\
\hline $17-70$ & 4531 & $Q$ & ${ }^{3} \mathrm{H}, \mathrm{NO}_{3}$ \\
\hline $19-43$ & 4417 & $Q$ & ${ }^{3} \mathrm{H}, \mathrm{NO}_{3}$ \\
\hline & & A & WQ \\
\hline
\end{tabular}

(a) Water quality analyses (designated $W Q$ ) include the following: $\mathrm{pH}$, Conductance, $\mathrm{Ca}, \mathrm{Mg}, \mathrm{Na}, \mathrm{CO}_{3}, \mathrm{HCO}_{3}, \mathrm{~K}, \mathrm{~B}$, $\mathrm{NO}_{3}-\mathrm{N}, \mathrm{Cl}, \mathrm{SO}_{4}-\mathrm{S}$, and dissolved solids. 
V. GROUND WATER (contd)

\begin{tabular}{|c|c|c|c|}
\hline Well Number & EMA\# & Frequency & Analyses (a) \\
\hline$(699-) 19-47 \mathrm{~A}$ & 4609 & $Q$ & ${ }^{3} \mathrm{H}, \quad \mathrm{NO}_{3}$ \\
\hline $19-58$ & 4528 & $Q$ & $\mathrm{NO}_{3}$ \\
\hline \multirow[t]{2}{*}{$19-88$} & 4522 & $Q$ & $\mathrm{NO}_{3}$ \\
\hline & & A & $F$ \\
\hline \multirow[t]{2}{*}{ 20-E5 A } & 4838 & $Q$ & ${ }^{3} \mathrm{H}, \mathrm{NO}_{3}, \mathrm{r}$ \\
\hline & & A & WQ \\
\hline 20-E5 P & 4705 & Q & ${ }^{3} \mathrm{H}, \quad \mathrm{NO}_{3}$ \\
\hline 20-E5 Q & 4706 & $Q$ & ${ }^{3} \mathrm{H}, \mathrm{NO}_{3}$ \\
\hline 20-E5 R & 4707 & $Q$ & ${ }^{3} \mathrm{H}, \mathrm{NO}_{3}$ \\
\hline $20-E 120$ & 4567 & $Q$ & ${ }^{3} \mathrm{H}, \mathrm{NO}_{3}$ \\
\hline $20-E 12 P$ & 4611 & $Q$ & ${ }^{3} \mathrm{H}, \mathrm{NO}_{3}$ \\
\hline $20-20$ & 4418 & $Q$ & $\mathrm{~B},{ }^{3} \mathrm{H}, \mathrm{NO}_{3}$, \\
\hline \multirow[t]{2}{*}{$20-39$} & 4559 & $Q$ & ${ }^{3} \mathrm{H}, \mathrm{NO}_{3}$ \\
\hline & & A & $\gamma$ \\
\hline \multirow[t]{2}{*}{$22-70$} & 4595 & Q & ${ }^{3} \mathrm{H}, \mathrm{NO}_{3}$ \\
\hline & & A & $\gamma$ \\
\hline $22-82$ & 4529 & $Q$ & ${ }^{3} \mathrm{H}, \mathrm{NO}_{3}$ \\
\hline $23-7$ & 4855 & M & ${ }^{3} \mathrm{H}, \quad \mathrm{NO}_{3}$ \\
\hline $24-1 P$ & 4710 & $Q$ & ${ }^{3} \mathrm{H}, \mathrm{NO}_{3}$ \\
\hline 24-1 Q & 4711 & $Q$ & ${ }^{3} \mathrm{H}, \mathrm{NO}_{3}$ \\
\hline $24-1 R$ & 4712 & $Q$ & ${ }^{3} \mathrm{H}, \mathrm{NO}_{3}$ \\
\hline 24-1 S & 4713 & $Q$ & ${ }^{3} \mathrm{H}, \quad \mathrm{NO}_{3}$ \\
\hline $24-1 T$ & 4764 & $Q$ & ${ }^{3} \mathrm{H}, \mathrm{NO}_{3}$ \\
\hline $24-33$ & 4416 & $Q$ & $\mathrm{~B},{ }^{3} \mathrm{H}, \mathrm{NO}_{3}, \gamma$ \\
\hline $24-46$ & 4525 & $Q$ & ${ }^{3} \mathrm{H}, \mathrm{NO}_{3}$ \\
\hline $25-55$ & 4415 & $Q$ & ${ }^{3} \mathrm{H}, \mathrm{NO}_{3}$ \\
\hline $25-70$ & 4452 & SA & ${ }^{3} \mathrm{H}, \mathrm{NO}_{3}$ \\
\hline & & A & WQ \\
\hline $26-15$ & 4518 & $Q$ & $\mathrm{~B},{ }^{3} \mathrm{H}, \mathrm{NO}_{3}$, \\
\hline & & A & WQ \\
\hline $26-89$ & 4598 & $S A$ & $\mathrm{NO}_{3}$ \\
\hline & & A & WQ \\
\hline $27-4$ & 4857 & M & ${ }^{3} \mathrm{H}, \mathrm{NO}_{3}$ \\
\hline
\end{tabular}

(a) Water quality analyses (designated $W Q$ ) include the following: $\mathrm{PH}$, Conductance, $\mathrm{Ca}, \mathrm{Mg}, \mathrm{Na}, \mathrm{CO}_{3}, \mathrm{HCO}_{3}, \mathrm{~K}, \mathrm{~B}$, $\mathrm{NO}_{3}-\mathrm{N}, \mathrm{Cl}, \mathrm{SO}_{4}-\mathrm{S}$, and dissolved solids. 
V. GROUND WATER (conto)

\begin{tabular}{|c|c|c|c|}
\hline We11 Number & EMA\# & Frequency & Analyses (a) \\
\hline$(699-) 27-8$ & 4557 & $Q$ & $\mathrm{~B},{ }^{3} \mathrm{H}, \mathrm{NO}_{3}, \mathrm{r}$ \\
\hline $29-78$ & 4594 & $Q$ & ${ }^{3} \mathrm{H}, \mathrm{NO}_{3}$ \\
\hline $28-400$ & 4481 & $Q$ & ${ }_{3} \mathrm{H}, \mathrm{NO}_{3}, \quad \mathrm{r}$ \\
\hline $28-40 P$ & 4754 & $Q$ & ${ }_{3}^{3} \mathrm{H}, \mathrm{NO}_{3}, \quad \mathrm{r}$ \\
\hline $28-52$ & 4521 & Q & ${ }^{3} \mathrm{H}, \mathrm{NO}_{3}, \mathrm{r}$ \\
\hline $31-310$ & 4471 & $Q$ & ${ }^{3} \mathrm{H}, \mathrm{NO}_{3}, \quad \mathrm{r}$ \\
\hline $31-31 P$ & 4738 & $Q$ & ${ }^{3} \mathrm{H}, \mathrm{NO}_{3}, \gamma$ \\
\hline $31-53 \quad B$ & 4520 & SA & ${ }^{3} \mathrm{H}, \mathrm{NO}_{3}, \gamma$ \\
\hline & & A & WQ \\
\hline $31-650$ & 4495 & $Q$ & ${ }^{3} \mathrm{H}, \mathrm{NO}_{3}$ \\
\hline $32-22$ & 4794 & $Q$ & $\mathrm{~B},{ }^{3} \mathrm{H}, \mathrm{NO}_{3}, \gamma$ \\
\hline $32-42$ & 4777 & Q & ${ }^{3} \mathrm{H}, \quad \mathrm{NO}_{3}$ \\
\hline $32-43$ & 4778 & $Q$ & ${ }^{3} \mathrm{H}, \mathrm{NO}_{3}, \mathrm{r}$ \\
\hline $32-62$ & 4550 & $Q$ & ${ }^{3} \mathrm{H}, \mathrm{NO}_{3}, \alpha$ \\
\hline $32-70$ & 4492 & $Q$ & $\mathrm{~B},{ }^{3} \mathrm{H}, \mathrm{NO}_{3}, \gamma$ \\
\hline $32-72$ & 4491 & Q & ${ }^{3} \mathrm{H}, \mathrm{NO}_{3}, \mathrm{r}$ \\
\hline $32-77$ & 4446 & $Q$ & $\mathrm{~B},{ }^{3} \mathrm{H}, \mathrm{NO}_{3}, \mathrm{r}$ \\
\hline $33-42$ & 4779 & $Q$ & $\mathrm{~B},{ }^{3} \mathrm{H}, \mathrm{NO}_{3}, \mathrm{r}$ \\
\hline $33-56$ & 4523 & Q & $\mathrm{B}, \alpha,{ }^{3} \mathrm{H}, \mathrm{NO}_{3}$, \\
\hline $34-39 \mathrm{~A}$ & 4448 & Q & $\mathrm{B},{ }^{3} \mathrm{H}, \mathrm{NO}_{3}, \gamma$ \\
\hline $34-41$ & 4789 & Q & ${ }^{3} \mathrm{H}, \mathrm{NO}_{3}, \gamma$ \\
\hline $34-42$ & 4790 & Q & $\mathrm{B},{ }^{3} \mathrm{H}, \mathrm{NO}_{3}, \mathrm{r}$ \\
\hline & & A & WQ \\
\hline $34-51$ & 4414 & $Q$ & $\mathrm{~B},{ }^{3} \mathrm{H}, \mathrm{NO}_{3}, \gamma$ \\
\hline $34-88$ & 4439 & Q & ${ }^{3} \mathrm{H}, \mathrm{NO}_{3}$ \\
\hline $35-9$ & 4419 & $Q$ & $\mathrm{~B},{ }^{3} \mathrm{H}, \mathrm{NO}_{3}, \mathrm{r}$ \\
\hline $35-66$ & 4494 & Q & $\mathrm{B},{ }^{3} \mathrm{H}, \mathrm{NO}_{3}, \gamma$ \\
\hline $35-70$ & 4441 & Q & $\mathrm{B},{ }^{3} \mathrm{H}, \mathrm{NO}_{3}, \gamma$ \\
\hline & & A & WQ \\
\hline $36-46 P$ & 4751 & Q & ${ }^{3} \mathrm{H}, \mathrm{NO}_{3}$, \\
\hline & & $S A$ & $\begin{array}{l}\gamma \\
3\end{array}$ \\
\hline $36-46 \mathrm{Q}$ & 4752 & Q & ${ }^{3} \mathrm{H}, \mathrm{NO}_{3}$, \\
\hline & & SA & $\gamma$ \\
\hline $36-61 \mathrm{~A}$ & 4447 & Q & $\mathrm{NO}_{3}$, \\
\hline
\end{tabular}

(a) Water quality analyses (designated $W Q$ ) include the following: $\mathrm{pH}$, Conductance, $\mathrm{Ca}, \mathrm{Mg}, \mathrm{Na}, \mathrm{CO}_{3}, \mathrm{HCO}_{3}, \mathrm{~K}, \mathrm{~B}$, $\mathrm{NO}_{3}-\mathrm{N}, \mathrm{Cl}, \mathrm{SO}_{4}-\mathrm{S}$, and dissolved solids. 
V. GROUND WATER (contd)

\begin{tabular}{|c|c|c|c|}
\hline We 11 Number & EMA\# & Frequency & Analyses (a) \\
\hline$(699-) 36-61$ B & 4549 & Q & \multirow{3}{*}{$\begin{array}{ll}{ }^{3} \mathrm{H}, & \mathrm{NO}_{3} \\
3_{\mathrm{H}}, & \mathrm{NO}_{3}\end{array}$} \\
\hline \multirow[t]{2}{*}{$36-93$} & 4579 & SA & \\
\hline & & A & \\
\hline $37-43$ & 4480 & $Q$ & \multirow{4}{*}{$\begin{array}{l}{ }^{\mathrm{B}},{ }^{3} \mathrm{H}, \mathrm{NO}_{3}, \mathrm{Y} \\
3_{\mathrm{H},} \mathrm{NO}_{3} \\
{ }^{3} \mathrm{H}, \mathrm{NO}_{3}\end{array}$} \\
\hline $37-82 A$ & 4554 & $Q$ & \\
\hline \multirow[t]{2}{*}{$38-65$} & 4546 & Q & \\
\hline & & SA & \\
\hline $38-70$ & 4493 & Q & \multirow{5}{*}{$\begin{array}{l}B,{ }^{3} \mathrm{H}, \mathrm{NO}_{3}, \\
\beta,{ }^{3} \mathrm{H}, \mathrm{NO}_{3}, \\
B,{ }^{3} \mathrm{H}, \mathrm{NO}_{3}, \\
\beta,{ }^{3} \mathrm{H}, \mathrm{NO}_{3}, \\
{ }^{3} \mathrm{H},\end{array}$} \\
\hline $39-1$ & 4871 & Q & \\
\hline $39-39$ & 4791 & SA & \\
\hline $39-79$ & 4444 & $Q$ & \\
\hline \multirow[t]{2}{*}{$40-1$} & 4566 & $Q$ & \\
\hline & & A & \multirow{3}{*}{$\begin{array}{l}Y, W Q \\
3_{\mathrm{H},} \mathrm{NO}_{3}\end{array}$} \\
\hline \multirow[t]{2}{*}{$40-33$} & 4431 & $Q$ & \\
\hline & & A & \\
\hline $40-62$ & 4458 & Q & \multirow{4}{*}{$\begin{array}{l}{ }^{3} \mathrm{H}, \mathrm{NO}_{3} \\
{ }^{3} \mathrm{H}, \mathrm{NO}_{3} \\
{ }^{3},{ }^{3} \mathrm{H}, \mathrm{NO}_{3}, \mathrm{r} \\
{ }^{3} \mathrm{H}, \mathrm{NO}_{3}\end{array}$} \\
\hline $41-1$ & 4858 & M & \\
\hline $41-23$ & 4430 & $Q$ & \\
\hline $42-2$ & 4859 & $M$ & \\
\hline $42-12$ & 4517 & Q & \multirow{3}{*}{$\begin{array}{l}{ }^{3},{ }^{3}, \mathrm{NO}_{3}, \mathrm{r} \\
{ }^{3} \mathrm{H}, \mathrm{NO}_{3}\end{array}$} \\
\hline $43-3$ & 4861 & $M$ & \\
\hline $43-88$ & 4836 & $Q$ & \\
\hline $44-4$ & 4872 & Q & \multirow{4}{*}{$\begin{array}{l}\mathrm{B},{ }^{3} \mathrm{H}, \mathrm{NO}_{3}, \gamma \\
{ }^{3} \mathrm{H}, \mathrm{NO} \\
3\end{array}$} \\
\hline $44-64$ & 4548 & $Q$ & \\
\hline $45-4$ & 4862 & $M$ & \\
\hline \multirow[t]{2}{*}{$45-42$} & 4450 & $Q$ & \\
\hline & & A & \multirow{8}{*}{$\begin{array}{l}\mathrm{WQ} \\
3_{\mathrm{H}, \mathrm{NO}} \\
\mathrm{B}, \mathrm{\gamma} \\
\mathrm{\beta}_{3},{ }^{\mathrm{H}, \mathrm{NO}_{3}, \mathrm{r}} \\
3_{\mathrm{H}, \mathrm{NO}_{3}}\end{array}$} \\
\hline \multirow[t]{2}{*}{$45-69$} & 4449 & Q & \\
\hline & & SA & \\
\hline $46-4$ & 4873 & Q & \\
\hline $46-5$ & 4863 & $M$ & \\
\hline $46-21$ & 4479 & Q & \\
\hline $47-6$ & 4864 & $M$ & \\
\hline $47-35$ & 4478 & $Q$ & \\
\hline
\end{tabular}

(a) Water quality analyses (designated $W Q$ ) include the following: $\mathrm{pH}$, Conductance, $\mathrm{Ca}, \mathrm{Mg}, \mathrm{Na}, \mathrm{CO}_{3}, \mathrm{HCO}_{3}, \mathrm{~K}, \mathrm{~B}$, $\mathrm{NO}_{3}-\mathrm{N}, \mathrm{Cl}, \mathrm{SO}_{4}-\mathrm{S}$, and dissolved solids. 
V. GROUND WATER (contd)

\begin{tabular}{|c|c|c|c|}
\hline We 11 Number & EMA\# & Frequency & Analyses (a) \\
\hline \multirow[t]{2}{*}{$(699-) 47-46$} & 4564 & $Q$ & \multirow{4}{*}{$\begin{array}{l}{ }^{3} \mathrm{H}, \mathrm{NO}_{3} \\
\mathrm{~B}, \mathrm{r} \\
3^{\mathrm{H}, \mathrm{NO}_{3}, \mathrm{Y}} \\
3_{\mathrm{H}, \mathrm{NO}_{3}}\end{array}$} \\
\hline & & $S A$ & \\
\hline $47-60$ & 4434 & Q & \\
\hline $48-7$ & 4756 & $Q$ & \\
\hline $48-18$ & 4830 & Q & ${ }_{3}^{3} \mathrm{H}, \mathrm{NO}_{3}$ \\
\hline $48-71$ & 4487 & $Q$ & ${ }^{3} \mathrm{H}, \mathrm{NO}_{3}$ \\
\hline \multirow[t]{2}{*}{$49-13$} & \multirow[t]{2}{*}{4771} & 0 & ${ }^{3} \mathrm{H}, \mathrm{NO}_{3}$ \\
\hline & & A & WQ \\
\hline $49-28$ & 4816 & $Q$ & \\
\hline $49-55$ & 4562 & $Q$ & $\mathrm{~B},{ }^{3} \mathrm{H}, \mathrm{NO}_{3}, \mathrm{Y}$ \\
\hline $49-57$ & 4485 & $Q$ & $\beta,{ }^{3} \mathrm{H}, \mathrm{NO}_{3}, \mathrm{r}$ \\
\hline \multirow[t]{2}{*}{$49-79$} & \multirow[t]{2}{*}{4443} & $Q$ & ${ }^{3} \mathrm{H}, \mathrm{NO}_{3}$ \\
\hline & & $A$ & WQ \\
\hline $50-19$ & 4776 & SA & $\mathrm{NO}_{3}$ \\
\hline \multirow[t]{2}{*}{$50-28 B$} & \multirow[t]{2}{*}{4844} & $Q$ & ${ }^{3} \mathrm{H}, \mathrm{NO}_{3}$ \\
\hline & & A & WQ \\
\hline $50-30$ & 4451 & $Q$ & $3_{\mathrm{H}, \mathrm{NO}_{3}}$ \\
\hline $50-42$ & 4460 & $Q$ & ${ }^{3} \mathrm{H}, \mathrm{NO}_{3}$ \\
\hline \multirow[t]{2}{*}{$50-53$} & \multirow[t]{2}{*}{4473} & $Q$ & $8,{ }^{3} \mathrm{H}, \mathrm{NO}_{3}, \mathrm{r}$ \\
\hline & & A & WQ \\
\hline $50-85$ & 4497 & Q & ${ }^{3} \mathrm{H}, \mathrm{NO}_{3}$ \\
\hline $51-63$ & 4488 & $Q$ & ${ }^{3} \mathrm{H}, \mathrm{NO}_{3}$ \\
\hline $51-75$ & 4496 & $Q$ & ${ }^{3} \mathrm{H}, \mathrm{NO}_{3}$ \\
\hline \multirow[t]{2}{*}{$53-35$} & \multirow[t]{2}{*}{4637} & $Q$ & ${ }^{3} \mathrm{H}, \mathrm{NO}_{3}$ \\
\hline & & A & $\gamma$ \\
\hline $53-103$ & 4772 & $Q$ & ${ }^{3} \mathrm{H}, \mathrm{NO}_{3}$ \\
\hline $54-34$ & 4638 & $Q$ & ${ }^{3} \mathrm{H}, \mathrm{NO}_{3}$ \\
\hline $54-37 \mathrm{~A}$ & 4853 & $Q$ & ${ }^{3} \mathrm{H}, \mathrm{NO}_{3}$ \\
\hline \multirow[t]{2}{*}{$54-42$} & \multirow[t]{2}{*}{4432} & $Q$ & $\mathrm{NO}_{3}$ \\
\hline & & SA & \\
\hline $54-45$ & 4811 & $Q$ & ${ }^{3} \mathrm{H}, \mathrm{NO}_{3}$ \\
\hline
\end{tabular}

(a) Water quality analyses (designated WQ) include the following: $\mathrm{pH}$, Conductance, $\mathrm{Ca}, \mathrm{Mg}, \mathrm{Na}, \mathrm{CO}_{3}, \mathrm{HCO}_{3}, \mathrm{~K}, \mathrm{~B}$, $\mathrm{NO}_{3}-\mathrm{N}, \mathrm{Cl}, \mathrm{SO}_{4}-\mathrm{S}$, and dissolved solids. 
V. GROUND WATER (contd)

\begin{tabular}{|c|c|c|c|}
\hline We 11 Number & EMA\# & Frequency & Analyses (a) \\
\hline$(699-) 54-57$ & 4469 & Q & \multirow{5}{*}{ 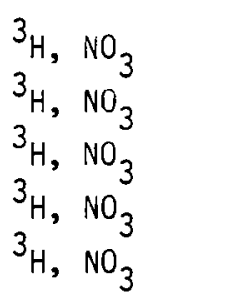 } \\
\hline $55-40$ & 4639 & $Q$ & \\
\hline $55-44$ & 4640 & $Q$ & \\
\hline $55-50 \mathrm{AO}$ & 4433 & $Q$ & \\
\hline \multirow[t]{3}{*}{$55-50 \mathrm{C}$} & \multirow[t]{3}{*}{4483} & $Q$ & \\
\hline & & SA & $\gamma$ \\
\hline & & A & \multirow{6}{*}{$\begin{array}{l}\mathrm{WQ} \\
3_{\mathrm{H}}, \mathrm{NO}_{3} \\
3_{\mathrm{H}}, \mathrm{NO}_{3} \\
\mathrm{NO}_{3} \\
\mathrm{NO}_{3} \\
3_{3}, \mathrm{NO}_{3} \\
3_{\mathrm{H}}, \mathrm{NO}_{3} \\
3 \mathrm{H}, \mathrm{NO}_{3}\end{array}$} \\
\hline $55-50 \quad D$ & 4484 & $Q$ & \\
\hline $55-70$ & 4442 & $Q$ & \\
\hline $\begin{array}{l}55-76 \\
55-89 \\
56-43\end{array}$ & $\begin{array}{l}4533 \\
4453 \\
4650\end{array}$ & $\begin{array}{l}Q \\
Q \\
Q\end{array}$ & \\
\hline $57-25 \mathrm{~A}$ & 4856 & $Q$ & \\
\hline $57-29 A$ & 4462 & $Q$ & \\
\hline $57-83$ & 4558 & Q & \multirow{5}{*}{$\begin{array}{l}\mathrm{NO}_{3} \\
3_{\mathrm{H}}, \mathrm{NO}_{3} \\
3_{\mathrm{H}}, \mathrm{NO}_{3} \\
3_{\mathrm{H}}, \mathrm{NO}_{3} \\
\mathrm{WQ}\end{array}$} \\
\hline $58-24$ & 4652 & Q & \\
\hline $59-32$ & 4815 & $Q$ & \\
\hline \multirow[t]{2}{*}{$59-58$} & \multirow[t]{2}{*}{4827} & $Q$ & \\
\hline & & A & \\
\hline $59-80 \quad B$ & 4437 & $Q$ & \multirow{4}{*}{$\begin{array}{ll}\mathrm{NO}_{3} & \\
3_{\mathrm{H}}, & \mathrm{NO}_{3} \\
3_{\mathrm{H}}, & \mathrm{NO}_{3} \\
3_{\mathrm{H}}, & \mathrm{NO}_{3}\end{array}$} \\
\hline $60-32$ & 4814 & $Q$ & \\
\hline $60-57$ & 4826 & $Q$ & \\
\hline $60-60$ & 4435 & $Q$ & \\
\hline $61-37$ & 4694 & $Q$ & \multirow{4}{*}{$\begin{array}{ll}\mathrm{NO}_{3} \\
3_{\mathrm{H}}, & \mathrm{NO}_{3} \\
3_{\mathrm{H}}, & \mathrm{NO}_{3} \\
3_{\mathrm{H},} & \mathrm{NO}_{3}\end{array}$} \\
\hline $61-41$ & 4653 & $Q$ & \\
\hline $61-62$ & 4825 & Q & \\
\hline $61-66$ & 4474 & $Q$ & \\
\hline $62-31$ & 4813 & $Q$ & \multirow{7}{*}{ 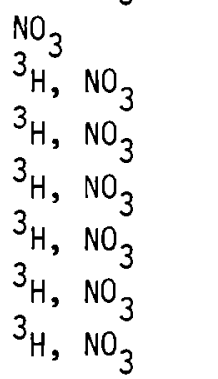 } \\
\hline $62-43 \mathrm{~F}$ & 4537 & $Q$ & \\
\hline $63-25$ & 4499 & $Q$ & \\
\hline $63-51$ & 4845 & SA & \\
\hline $63-55$ & 4823 & Q & \\
\hline $63-58$ & 4822 & Q & \\
\hline $63-90$ & 4436 & $Q$ & \\
\hline
\end{tabular}

(a) Water quality analyses (designated WQ) include the following: $\mathrm{pH}$, Conductance, $\mathrm{Ca}, \mathrm{Mg}, \mathrm{Na}, \mathrm{CO}_{3}, \mathrm{HCO}_{3}, \mathrm{~K}, \mathrm{~B}$, $\mathrm{NO}_{3}-\mathrm{N}, \mathrm{Cl}, \mathrm{SO}_{4}-\mathrm{S}$, and dissolved solids. 
V. GROUND WATER (contd)

\begin{tabular}{|c|c|c|c|}
\hline We 11 Number & EMA\# & Frequency & Analyses (a) \\
\hline \multirow[t]{3}{*}{$(699-) 64-27$} & \multirow[t]{3}{*}{4599} & $Q$ & \multirow{2}{*}{$\mathrm{NO}_{\mathrm{H}}{ }^{3}$} \\
\hline & & SA & \\
\hline & & A & \multirow{2}{*}{$\begin{array}{l}\text { WQ } \\
3_{H}\end{array}$} \\
\hline $64-62$ & 4824 & $Q$ & \\
\hline $65-23$ & 4851 & SA & \multirow{6}{*}{$\begin{array}{ll}{ }^{3} \mathrm{H}, & \mathrm{NO}_{3}^{3} \\
3^{3} \mathrm{H}, & \mathrm{NO}_{3} \\
3^{3} \mathrm{H}, & \mathrm{NO}_{3} \\
{ }^{3} \mathrm{H}, & \mathrm{NO}_{3} \\
3^{3} \mathrm{H}, & \mathrm{NO}_{3} \\
3 \mathrm{H}, & \mathrm{NO}_{3}\end{array}$} \\
\hline $65-38$ & 4716 & $Q$ & \\
\hline $65-50$ & 4477 & Q & \\
\hline $65-59$ & 4532 & $Q$ & \\
\hline $65-72$ & 4468 & Q & \\
\hline $65-83$ & 4775 & Q & \\
\hline $66-23$ & 4547 & Q & $\mathrm{NO}_{3}$ \\
\hline $66-38$ & 4586 & Q & \multirow{4}{*}{$\begin{array}{l}\mathrm{NO}_{3} \\
\mathrm{NO}_{3} \\
3_{\mathrm{H}} \mathrm{NO}_{3} \\
3_{\mathrm{H},}, \mathrm{NO}_{3}\end{array}$} \\
\hline $66-39$ & 4812 & $Q$ & \\
\hline $66-58$ & 4821 & $Q$ & \\
\hline $66-64$ & 4820 & $Q$ & \\
\hline & & A & \multirow{3}{*}{${ }^{\mathrm{WQ}} \mathrm{H}, \mathrm{NO}_{3}$} \\
\hline $66-103$ & 4587 & SA & \\
\hline & & A & \\
\hline $67-51$ & 4561 & Q & \multirow{4}{*}{$\begin{array}{ll}3_{\mathrm{H}}, \mathrm{NO}_{3} \\
3_{\mathrm{H}}, \mathrm{NO}_{3} \\
3_{\mathrm{H},} \mathrm{NO}_{3} \\
3_{\mathrm{H},} \mathrm{NO}_{3}\end{array}$} \\
\hline $67-86$ & 4585 & $Q$ & \\
\hline $67-98$ & 4556 & Q & \\
\hline $68-105$ & 4588 & SA & \\
\hline & & A & \multirow{6}{*}{$\begin{array}{ll}\mathrm{y} \\
3_{\mathrm{H}}, & \mathrm{NO}_{3} \\
3_{\mathrm{H}}, & \mathrm{NO}_{3} \\
3_{\mathrm{H}}, & \mathrm{NO}_{3} \\
3^{3} \mathrm{H}, & \mathrm{NO}_{3} \\
3_{\mathrm{H}}, & \mathrm{NO}_{3}\end{array}$} \\
\hline $69-38$ & 4461 & Q & \\
\hline $70-68$ & 4455 & SA & \\
\hline $71-30$ & 4490 & SA & \\
\hline $71-52$ & 4454 & $Q$ & \\
\hline $71-77$ & 4584 & SA & \\
\hline & & A & \multirow{5}{*}{$\begin{array}{l}\mathrm{WQ} \\
3_{\mathrm{H}}, \mathrm{NO}_{3} \\
3_{\mathrm{H}}, \mathrm{NO}_{3} \\
\mathrm{WQ} \\
3_{\mathrm{H},} \mathrm{NO}_{3}\end{array}$} \\
\hline $72-73$ & 4569 & SA & \\
\hline \multirow[t]{2}{*}{$72-88$} & 4465 & Q & \\
\hline & & A & \\
\hline $72-92$ & 4565 & SA & \\
\hline
\end{tabular}

(a) Water quality analyses (designated WQ) include the following: $\mathrm{pH}$, Conouctance, $\mathrm{Ca}, \mathrm{Mg}, \mathrm{Na}, \mathrm{CO}_{3}, \mathrm{HCO}_{3}, \mathrm{~K}, \mathrm{~B}$, $\mathrm{NO}_{3}-\mathrm{N}, \mathrm{Cl}, \mathrm{SO}_{4}-\mathrm{S}$, and dissolved solids. 
V. GROUND WATER (contd)

\begin{tabular}{|c|c|c|c|}
\hline We 11 Number & EMA\# & Frequency & Analyses (a) \\
\hline$(699-) 72-98$ & 4463 & $S A$ & $3_{\mathrm{H}, \mathrm{NO}}$ \\
\hline $73-61$ & 4583 & $Q$ & ${ }^{3} \mathrm{H}, \mathrm{NO}_{3}$ \\
\hline $74-44$ & 4516 & $Q$ & ${ }^{3} \mathrm{H}, \mathrm{NO}_{3}$ \\
\hline & & A & WQ \\
\hline $74-60$ & 4583 & SA & $\mathrm{NO}_{3}$ \\
\hline $77-36$ & 4500 & $Q$ & $3_{\mathrm{H}, \mathrm{NO}_{3}}$ \\
\hline $77-54$ & 4512 & $Q$ & $\mathrm{NO}_{3}$ \\
\hline $78-62$ & 4511 & SA & $\mathrm{NO}_{3}$ \\
\hline & & A & WQ \\
\hline $80-43 P$ & 4760 & SA & $\mathrm{NO}_{3}$ \\
\hline $80-43 Q$ & 4761 & SA & $\mathrm{NO}_{3}$ \\
\hline $80-43 R$ & 4762 & SA & $\mathrm{NO}_{3}$ \\
\hline $80-43 \mathrm{~S}$ & 4763 & SA & $\mathrm{NO}_{3}$ \\
\hline $81-58$ & 4597 & Q & ${ }^{3} \mathrm{H}, \mathrm{NO}_{3}$ \\
\hline & & $A$ & WQ \\
\hline $83-47$ & 4515 & SA & $3_{H}$ \\
\hline $84-35$ A 0 & 4596 & SA & ${ }^{3} \mathrm{H}, \mathrm{NO}_{3}$ \\
\hline $87-55$ & 4792 & Q & ${ }^{3} \mathrm{H}, \mathrm{NO}_{3}$ \\
\hline & & $A$ & WQ \\
\hline $89-35$ & 4571 & $Q$ & $\mathrm{NO}_{3}$ \\
\hline & & A & WQ \\
\hline $90-45$ & 4770 & $Q$ & ${ }^{3} \mathrm{H}$ \\
\hline & & SA & $\mathrm{NO}_{3}$ \\
\hline $96-49$ & 4591 & $Q$ & ${ }^{3} \mathrm{H}, \mathrm{NO}_{3}$ \\
\hline $97-43$ & 4590 & $Q$ & ${ }^{3} \mathrm{H}, \mathrm{NO}_{3}$ \\
\hline $97-51 \mathrm{~A}$ & 4728 & $Q$ & ${ }^{3} \mathrm{H}, \mathrm{NO}_{3}$ \\
\hline $101-48 B$ & 4846 & SA & ${ }^{3} \mathrm{H}, \mathrm{NO}_{3}$ \\
\hline & $\underline{100 \mathrm{~A}}$ & Wells & \\
\hline (199-)B3-1 & 1851 & $S A$ & $3_{H}$ \\
\hline$B 3-2 P$ & 1856 & SA & ${ }^{3} \mathrm{H}, \mathrm{NO}_{3}$ \\
\hline$B 3-2 Q$ & 1857 & SA & \\
\hline B4-1 & 1853 & $Q$ & ${ }^{3} \mathrm{H}, \mathrm{NO}_{3}$ \\
\hline B4-2 & 1854 & $Q$ & ${ }^{3} \mathrm{H}, \mathrm{NO}_{3}$ \\
\hline
\end{tabular}

(a) Water quality analyses (designated WQ) include the following: $\mathrm{pH}$, Conductance, $\mathrm{Ca}, \mathrm{Mg}, \mathrm{Na}, \mathrm{CO}_{3}, \mathrm{HCO}_{3}, \mathrm{~K}, \mathrm{~B}$, $\mathrm{NO}_{3}-\mathrm{N}, \mathrm{Cl}, \mathrm{SO}_{4}-\mathrm{S}$, and dissolved solids. 
v. GROUND WATER (contd)

\begin{tabular}{|c|c|c|c|}
\hline We 11 Number & EMA\# & Frequency & Analyses (a) \\
\hline (199-)84-3 & 1855 & $Q$ & ${ }^{3} \mathrm{H}, \mathrm{NO}_{3}$ \\
\hline B4-4 & 1891 & SA & $\mathrm{B},{ }^{3} \mathrm{H}, \mathrm{NO}_{3} \mathrm{r}$ \\
\hline B5-1 & 1895 & Q & ${ }^{3} \mathrm{H}, \mathrm{NO}_{3}$ \\
\hline 89-1 & 1893 & SA & ${ }^{3} \mathrm{H}, \mathrm{NO}_{3}$ \\
\hline D2-5 & 1894 & Q & ${ }^{3} \mathrm{H}, \mathrm{NO}_{3}$ \\
\hline D5-12 & 1892 & $Q$ & $\mathrm{~B},{ }^{3} \mathrm{H}, \mathrm{NO}_{3} \gamma$ \\
\hline D8-3 & 1862 & $Q$ & ${ }^{3} \mathrm{H}, \mathrm{NO}_{3}$ \\
\hline F5-1 & 1865 & $Q$ & ${ }^{3} \mathrm{H}, \mathrm{NO}_{3}$ \\
\hline F5-3 & 1867 & $Q$ & ${ }^{3} \mathrm{H}, \quad \mathrm{NO}_{3}$ \\
\hline$F 5-4$ & 1868 & Q & ${ }^{3} \mathrm{H}, \mathrm{NO}_{3}$ \\
\hline F5-6 & 1870 & $Q$ & ${ }^{3} \mathrm{H}, \mathrm{NO}_{3}$ \\
\hline$F 7-1$ & 1871 & Q & ${ }^{3} \mathrm{H}, \mathrm{NO}_{3}$ \\
\hline F8-1 & 1888 & $Q$ & ${ }_{3}^{3} \mathrm{H}, \mathrm{NO}_{3}$ \\
\hline F8-2 & 1889 & Q & ${ }_{3}^{3} \mathrm{H}, \mathrm{NO}_{3}$ \\
\hline H3-1 & 1890 & $Q$ & ${ }^{3} \mathrm{H}, \mathrm{NO}_{3}$ \\
\hline $\mathrm{H} 4-3$ & 1877 & M & $\begin{array}{l}\mathrm{B}, \mathrm{U}, \mathrm{H}, \mathrm{NO}_{3}, \mathrm{Cr}^{+6} \\
\mathrm{~F}, \mathrm{Cu}\end{array}$ \\
\hline & & Q & $\gamma$ \\
\hline & & A & WQ \\
\hline$k-11$ & 1882 & $\begin{array}{l}Q \\
S A\end{array}$ & ${ }_{\gamma}^{3} \mathrm{H}, \mathrm{NO}_{3}$ \\
\hline$k-19$ & 1884 & $Q$ & ${ }^{3} \mathrm{H}, \mathrm{NO}_{3}$ \\
\hline$k-20$ & 1885 & Q & ${ }_{3}^{3} \mathrm{H}, \mathrm{NO}_{3}$ \\
\hline$k-22$ & 1887 & Q & ${ }^{3} \mathrm{H}, \mathrm{NO}_{3}$ \\
\hline$k-27$ & 1911 & $Q$ & ${ }_{2}^{3} \mathrm{H}, \mathrm{r}$ \\
\hline$K-28$ & 1912 & $Q$ & ${ }^{3} \mathrm{H}, \mathrm{r}$ \\
\hline$k-29$ & 1913 & Q & ${ }^{3} \mathrm{H}, \mathrm{\gamma}$ \\
\hline$k-30$ & 1914 & $Q$ & ${ }_{2}^{3} \mathrm{H}, \mathrm{r}$ \\
\hline$N-1$ & 1908 & $\begin{array}{l}Q \\
S A\end{array}$ & $\begin{array}{l}{ }_{\mathrm{H}} \mathrm{H}, \mathrm{NO}_{3}, \mathrm{r} \\
\mathrm{Sr}\end{array}$ \\
\hline $\mathrm{N}-2$ & 1904 & $\begin{array}{l}Q \\
S A\end{array}$ & $\begin{array}{l}{ }^{3} \mathrm{H}, \mathrm{NO}_{3}, r \\
\mathrm{Sr}\end{array}$ \\
\hline $\mathrm{N}-30$ & 1896 & $\begin{array}{l}Q \\
S A\end{array}$ & $\begin{array}{l}B,{ }^{3} H, r \\
S r\end{array}$ \\
\hline$N-4$ & 1899 & $\begin{array}{l}Q \\
S A\end{array}$ & $\begin{array}{l}\mathrm{B},{ }^{3} \mathrm{H}, \mathrm{NO}_{3}, \mathrm{r} \\
\mathrm{Sr}\end{array}$ \\
\hline $\mathrm{N}-5$ & 1909 & $\begin{array}{l}Q \\
S A\end{array}$ & $\begin{array}{l}{ }_{\mathrm{H}}, \mathrm{NO}_{3}, r \\
\mathrm{Sr}\end{array}$ \\
\hline
\end{tabular}

(a) Water quality analyses (designated WQ) include the following: $\mathrm{pH}$, Conductance, $\mathrm{Ca}, \mathrm{Mg}, \mathrm{Na}, \mathrm{CO}_{3}, \mathrm{HCO}_{3}, \mathrm{~K}, \mathrm{~B}$, $\mathrm{NO}_{3}-\mathrm{N}, \mathrm{Cl}, \mathrm{SO}_{4}-\mathrm{S}$, and dissolved solids. 
V. GROUND WATER (contd)

\begin{tabular}{|c|c|c|c|}
\hline Well Number & EMA\# & Frequency & Analyses (a) \\
\hline$(199-) N-6$ & 1901 & Q & $B,{ }^{3} H, \gamma$ \\
\hline & & SA & Sr \\
\hline $\mathrm{N}-7$ & 1910 & $\begin{array}{l}Q \\
S A\end{array}$ & $\begin{array}{l}{ }_{\mathrm{H}}^{\mathrm{H}}, \mathrm{NO}_{3}, \mathrm{r} \\
\mathrm{Sr}\end{array}$ \\
\hline $\mathrm{N}-10 \mathrm{P}$ & 1900 & $\begin{array}{l}Q \\
S A\end{array}$ & $\begin{array}{l}\mathrm{B},{ }^{3} \mathrm{H}, \mathrm{NO}_{3}, \mathrm{r} \\
\mathrm{Sr}\end{array}$ \\
\hline$N-14$ & 1902 & $\begin{array}{l}Q \\
S A\end{array}$ & $\begin{array}{l}\mathrm{B},{ }^{3} \mathrm{H}, \mathrm{NO}_{3}, \mathrm{r} \\
\mathrm{Sr}\end{array}$ \\
\hline$N-15$ & 1903 & $\begin{array}{l}Q \\
S A\end{array}$ & $\begin{array}{l}\mathrm{B},{ }^{3} \mathrm{H}, \mathrm{NO}_{3}, \mathrm{r} \\
\mathrm{Sr}\end{array}$ \\
\hline
\end{tabular}

300 Area Wells

\begin{tabular}{|c|c|c|c|}
\hline$(399-) 1-1$ & 4403 & Q & $\mathrm{B}, \mathrm{NO}_{3}, \mathrm{U}, \mathrm{Cr}^{+6}, \mathrm{~F}, \mathrm{r}$ \\
\hline $1-2$ & 4404 & Q & $\mathrm{B}, \mathrm{NO}_{3}, \mathrm{U}, \mathrm{Cr}^{+6}, \mathrm{~F}, \mathrm{r}$ \\
\hline $1-3$ & 4406 & Q & $\mathrm{B}, \mathrm{NO}_{3}, \mathrm{U}, \mathrm{Cr}^{+6}, \mathrm{~F}, \mathrm{r}$ \\
\hline $1-4$ & 4407 & 0 & $\mathrm{~B}, \mathrm{NO}_{3}, \mathrm{U}, \mathrm{Cr}^{+6}, \mathrm{~F}, \mathrm{r}$ \\
\hline $1-5$ & 4806 & M & $\beta, \mathrm{NO}_{3}, \mathrm{U}, \mathrm{Cr}^{+6}, \mathrm{~F}, \gamma$ (TO HEDL) \\
\hline $1-6$ & 4837 & Q & $\mathrm{B}, \mathrm{NO}_{3}, \mathrm{U}, \mathrm{Cr}^{+6}, \mathrm{~F}, \mathrm{\gamma}$ \\
\hline $2-1$ & 4402 & Q & $\mathrm{B}, \mathrm{NO}_{3}, \mathrm{U}, \mathrm{Cr}^{+6}, \mathrm{~F}, \mathrm{\gamma}$ \\
\hline $2-2$ & 4633 & Q & $\mathrm{B}, \mathrm{NO}_{3}, \mathrm{U}, \mathrm{Cr}^{+6}, \mathrm{~F}, \mathrm{\gamma}$ \\
\hline $2-3$ & 4634 & Q & $\beta, \mathrm{NO}_{3}, \mathrm{U}, \mathrm{Cr}^{+6}, \mathrm{~F}, \mathrm{\gamma}$ \\
\hline $3-1$ & 4401 & $Q$ & $\mathrm{~B}, \mathrm{NO}_{3}, \mathrm{U}, \mathrm{Cr}^{+6}, \mathrm{~F}, \mathrm{\gamma}$ \\
\hline $3-2$ & 3303 & M & $\mathrm{B}, \mathrm{NO}_{3}, \mathrm{U}, \mathrm{Cr}^{+6}, \mathrm{~F}, \mathrm{r}$ \\
\hline $3-3$ & 3034 & M & $\mathrm{B}, \mathrm{NO}_{3}, \mathrm{U}, \mathrm{Cr}^{+6}, \mathrm{~F}, \mathrm{r}$ \\
\hline $3-6$ & 3031 & M & $\mathrm{B}, \mathrm{NO}_{3}, \mathrm{U}, \mathrm{Cr}^{+6}, \mathrm{~F}, \mathrm{r}$ \\
\hline $3-7$ & 4839 & $M$ & $\beta, \mathrm{NO}_{3}, \mathrm{Cr}^{+6}, U, F, \gamma$ \\
\hline $3-9$ & 4626 & M & $\beta, \mathrm{NO}_{3}, U, \mathrm{Cr}^{+6}, \mathrm{~F}, \gamma, \mathrm{Sr}$ \\
\hline $3-10$ & 4627 & M & $\mathrm{B}, \mathrm{NO}_{3}, \mathrm{U}, \mathrm{Cr}^{+6}, \mathrm{~F}, \mathrm{r}, \mathrm{Sr}$ \\
\hline $3-11$ & 4628 & M & $\mathrm{B} \mathrm{NO}_{3}, \mathrm{U}, \mathrm{Cr}^{+6}, \mathrm{~F}, \gamma, \mathrm{Sr}$ \\
\hline $3-12$ & 4870 & M & $\mathrm{B}, \mathrm{NO}_{3}, \mathrm{U}, \mathrm{Cr}^{+6}, \mathrm{~F}, \mathrm{\gamma}$ \\
\hline $4-1$ & 4410 & $Q$ & $\mathrm{~B}, \mathrm{NO}_{3}, \mathrm{U}, \mathrm{Cr}^{+6}, \mathrm{~F}, \mathrm{r}$ \\
\hline $4-7$ & 4568 & $Q$ & $\mathrm{~B}, \mathrm{NO}_{3}, \mathrm{U}, \mathrm{Cr}^{+6}, \mathrm{~F}, \mathrm{r}$ \\
\hline $4-9$ & 4629 & M & $\mathrm{B}, \mathrm{NO}_{3}, \mathrm{U}, \mathrm{Cr}^{+6}, \mathrm{~F}, \mathrm{r}, \mathrm{Sr}$ \\
\hline $4-10$ & 4630 & Q & $\mathrm{B}, \mathrm{NO}_{3}, \mathrm{U}, \mathrm{Cr}^{+6}, \mathrm{~F}, \gamma, \mathrm{Sr}$ \\
\hline $5-1$ & 4411 & $Q$ & $\mathrm{~B}, \mathrm{NO}_{3}, \mathrm{U}, \mathrm{Cr}^{+6}, \mathrm{~F}, \mathrm{r}$ \\
\hline $6-1$ & 4409 & $Q$ & $\mathrm{~B}, \mathrm{NO}_{3}, \mathrm{U}, \mathrm{Cr}^{+6}, \mathrm{~F}, \mathrm{\gamma}$ \\
\hline
\end{tabular}

(a) Water quality analyses (designated WQ) include the following: $\mathrm{pH}$, Concuctance, $\mathrm{Ca}, \mathrm{Mg}, \mathrm{Na}, \mathrm{CO}_{3}, \mathrm{HCO}_{3}, \mathrm{~K}, \mathrm{~B}$, $\mathrm{NO}_{3}-\mathrm{N}, \mathrm{Cl}, \mathrm{SO}_{4}-\mathrm{S}$, and dissolved solids. 
V. GROUND WATER (contd)

\begin{tabular}{|c|c|c|c|}
\hline We 11 Number & EMA\# & Frequency & Analyses (a) \\
\hline$(399-) 8-1$ & 4405 & $Q$ & $\mathrm{~B}, \mathrm{NO}_{3}, \mathrm{U}, \mathrm{Cr}^{+6}, \mathrm{~F}, \mathrm{r}$ \\
\hline $8-2$ & 4408 & $Q$ & $8, \mathrm{NO}_{3}, \mathrm{U}, \mathrm{Cr}^{+6}, \mathrm{~F}, \mathrm{r}$ \\
\hline $8-3$ & 4412 & $Q$ & $\mathrm{~B}, \mathrm{NO}_{3}, \mathrm{U}, \mathrm{Cr}^{+6}, \mathrm{~F}, \mathrm{r}$ \\
\hline $8-4$ & 4865 & M & $\mathrm{B}, \mathrm{NO}_{3}, \mathrm{U}, \mathrm{Cr}^{+6}, \mathrm{~F}, \mathrm{r}$ \\
\hline
\end{tabular}

200 Area Wells

\begin{tabular}{|c|c|c|c|}
\hline$(299-)$ E19-1 & 2359 & $Q$ & $\mathrm{~B},{ }^{3} \mathrm{H}, \mathrm{NO}_{3}, \mathrm{Y}$ \\
\hline E23-1 & 2553 & $Q$ & $\mathrm{~B},{ }^{3} \mathrm{H}, \mathrm{NO}_{3}, \gamma$ \\
\hline E24-7 & 2542 & $Q$ & $\mathrm{~B},{ }^{3} \mathrm{H}, \mathrm{NO}_{3}, \mathrm{Y}$ \\
\hline$E 25-2$ & 2554 & $Q$ & $\mathrm{~B},{ }^{3} \mathrm{H}, \mathrm{NO}_{3}, \mathrm{r}$ \\
\hline E26-1 & 2545 & $Q$ & $\mathrm{~B},{ }^{3} \mathrm{H}, \mathrm{NO}_{3}, \mathrm{\gamma}$ \\
\hline$E 26-3$ & 2365 & $Q$ & $\mathrm{~B},{ }^{3} \mathrm{H}, \mathrm{NO}_{3}, \mathrm{r}$ \\
\hline$E 27-1$ & 2287 & $Q$ & $\mathrm{~B},{ }^{3} \mathrm{H}, \mathrm{NO}_{3}, \mathrm{Y}$ \\
\hline E28-1 & 2555 & 0 & $\mathrm{~B},{ }^{3} \mathrm{H}, \mathrm{NO}_{3}, \mathrm{\gamma}$ \\
\hline$E 28-5$ & 2285 & $Q$ & $\mathrm{~B},{ }^{3} \mathrm{H}, \mathrm{NO}_{3}, \mathrm{r}$ \\
\hline E33-14 & 2297 & $Q$ & $\mathrm{~B},{ }^{3} \mathrm{H}, \mathrm{NO}_{3}, \mathrm{Y}$ \\
\hline E34-1 & 2549 & $M$ & $\mathrm{NO}_{3}$ \\
\hline W6-1 & 2990 & $Q$ & $\mathrm{~B},{ }^{3} \mathrm{H}, \mathrm{NO}_{3}, \mathrm{Y}$ \\
\hline$W 10-5$ & 2890 & $Q$ & $\mathrm{~B},{ }^{3} \mathrm{H}, \mathrm{NO}_{3}, \mathrm{r}$ \\
\hline$w 11-9$ & 2881 & $Q$ & $\mathrm{~B},{ }^{3} \mathrm{H}, \mathrm{NO}_{3}, \mathrm{Y}$ \\
\hline W12-1 & 2883 & $Q$ & $\mathrm{~B},{ }^{3} \mathrm{H}, \mathrm{NO}_{3}, \mathrm{\gamma}$ \\
\hline$w 15-2$ & 2891 & $Q$ & $\mathrm{~B},{ }^{3} \mathrm{H}, \mathrm{NO}_{3}, \mathrm{Y}$ \\
\hline W18-3 & 3011 & $Q$ & $\mathrm{~B},{ }^{3} \mathrm{H}, \mathrm{NO}_{3}, \mathrm{r}$ \\
\hline W19-4 & 2938 & $Q$ & $\mathrm{~B},{ }^{3} \mathrm{H}, \mathrm{NO}_{3}, \mathrm{r}$ \\
\hline W21-1 & 2930 & $Q$ & $\mathrm{~B},{ }^{3} \mathrm{H}, \mathrm{NO}_{3}, \gamma$ \\
\hline W22-7 & 3014 & $Q$ & $\mathrm{~B},{ }_{3}^{3} \mathrm{H}, \mathrm{NO}_{3}, \mathrm{r}$ \\
\hline W22-9 & 3013 & $Q$ & $\mathrm{~B},{ }^{3} \mathrm{H}, \mathrm{NO}_{3}, \mathrm{\gamma}$ \\
\hline W22-10 & 2906 & $Q$ & $\alpha, \beta, S r, \gamma$ \\
\hline
\end{tabular}

(a) Water quality analyses (designated WQ) include the following: $\mathrm{pH}$, Conductance, $\mathrm{Ca}, \mathrm{Mg}, \mathrm{Na}, \mathrm{CO}_{3}, \mathrm{HCO}_{3}, \mathrm{~K}, \mathrm{~B}$, $\mathrm{NO}_{3}-\mathrm{N}, \mathrm{Cl}, \mathrm{SO}_{4}-\mathrm{S}$, and dissolved solids. 
VI. FOODSTUFFS

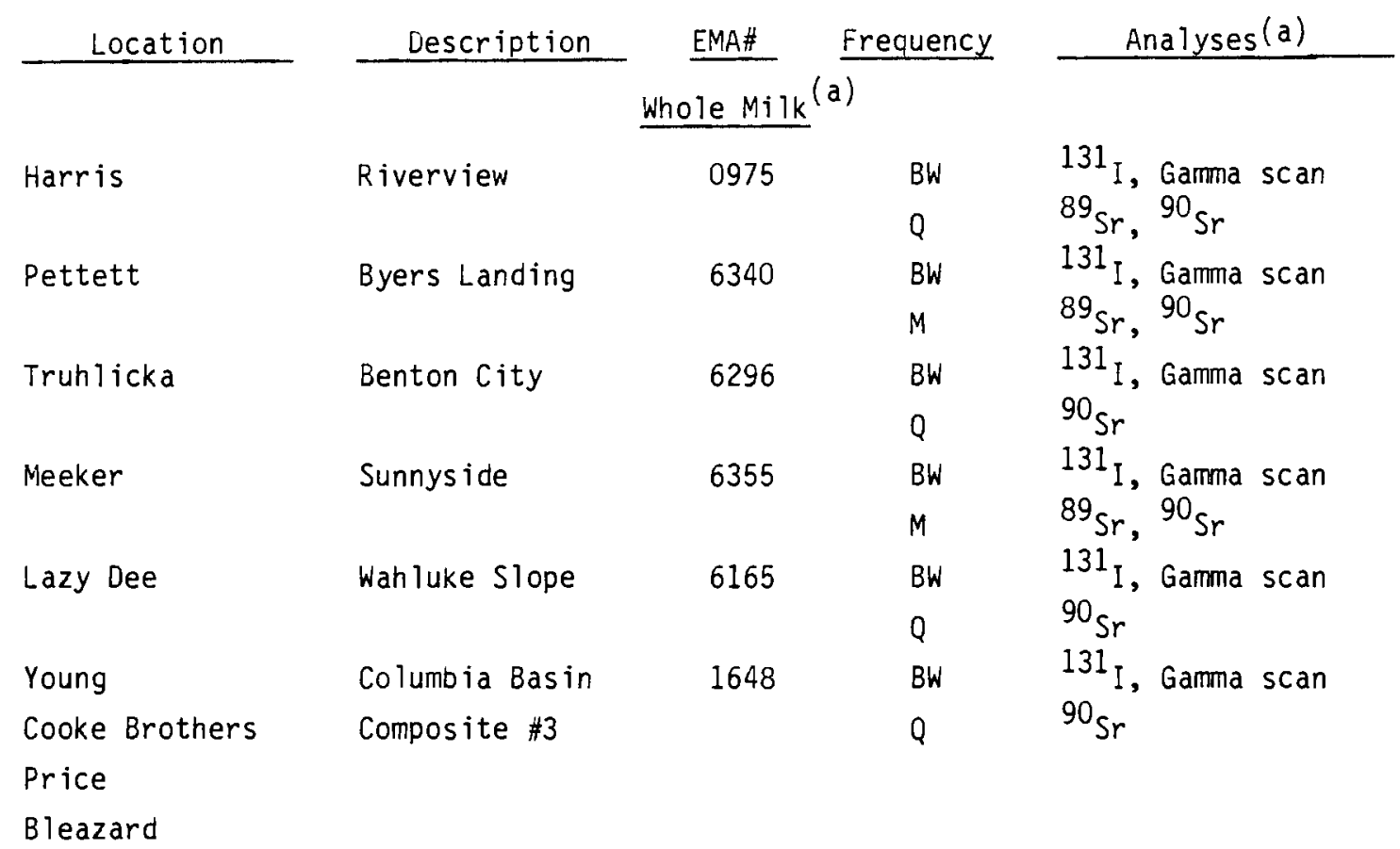

Meat

$\begin{array}{lllll}\text { Harris } & \text { Riverview } & 1292 & \text { SA } & \text { Gamma scan, }{ }^{90} \text { Sr } \\ \text { Local Farm } & \text { FFTF F Sector } & 6418 & \text { A } & \text { Gamma scan } \\ \text { Local Farm } & \text { Horn Rapids } & 6516 & \text { A } & \text { Gamma scan }\end{array}$

\section{Produce (Leafy Vegetables) $^{(b)}$}

\begin{tabular}{|c|c|c|c|c|c|}
\hline Kinne & Ringold & 6373 & A & Gamma & scan, \\
\hline Harris & Riverview & 1609 & M & Gamma & scan, \\
\hline Local Farm & Sunnyside & 6372 & A & Gamma & scan \\
\hline Local Farm & Benton City & 1612 & $B W$ & Gamma & scan, \\
\hline Local Farm & Sagemoor Vicinity & 6419 & A & Gamma & scan \\
\hline Local $\operatorname{Farm}^{(c)}$ & Walla Walla & 6409 & M & Gamma & scan \\
\hline Local Farm (c) & Moses Lake & 6407 & $M$ & Gamma & scan \\
\hline Local $F_{a r m}^{(c)}$ & MCNary & 6410 & M & Gamma & scan \\
\hline Local Farm $(c)$ & Othe 110 & 6408 & $M$ & Gamma & scan \\
\hline
\end{tabular}

(a) Sample of forage also collected but not analyzed unless a positive result for

(b) Sample collected only during growing season, May to October.

(c) Sample only from one source each month. 
VI. FOODSTUFFS (contd)

\begin{tabular}{|c|c|c|c|c|}
\hline Location & Description & EMA\# & Frequency & Analyses \\
\hline & & Farm Fr & & \\
\hline Sagemoor Vicinity & Peaches & 0567 & A & Gamma scan \\
\hline Sagemoor Vicinity & Plums & 0577 & A & Gamma scan \\
\hline Sagemoor Vicinity & Grapes & 6417 & A & Garmma scan \\
\hline Sagemoor Vicinity & Apples & 0565 & A & Gamma scan \\
\hline Sagemoor Vicinity & Pears & 6424 & A & Gamma scan \\
\hline Sagemoor Vicinity & Cherries & 0543 & A & Gamma scan \\
\hline Sunnyside & Cherries & 6453 & A & Gamma scan \\
\hline Sunnyside & Peaches & 6443 & A & Gamma scan \\
\hline Sunnyside & Plums & 6442 & A & Gamma scan \\
\hline Sunnyside & Pears & 6441 & A & Gamma scan \\
\hline Sunnyside & Apples & 6440 & A & Gamma scan \\
\hline Sunnyside & Grapes & 6454 & A & Gamma scan \\
\hline
\end{tabular}


VII. WILDLIFE

\begin{tabular}{|c|c|c|c|c|}
\hline Location & EMA\# & Species & Frequency & Analyses \\
\hline \multicolumn{5}{|c|}{$\underline{F i s h}^{(a)}$} \\
\hline Above Priest Rapids Dam & & $\begin{array}{l}\text { Mtn. Whitefish } \\
\text { Smallmouth Bass }\end{array}$ & $\begin{array}{l}\text { SA (April \& Oct.) } \\
\text { A (April) }\end{array}$ & $\begin{array}{l}\text { Gamma Scan } \\
\text { Gamma Scan }\end{array}$ \\
\hline Vernita to Priest Rapids & & Mtn. Whitefish & SA (April \& Oct.) & Gamma Scan \\
\hline $100-D$ & & Mtn. Whitefish & Q & Gamma Scan \\
\hline Hanford Slough & & $\begin{array}{l}\text { Mtn. Whitefish } \\
\text { Smal lmouth Bass }\end{array}$ & $\begin{array}{l}\text { SA (April \& Oct.) } \\
\text { A (April) }\end{array}$ & $\begin{array}{l}\text { Gamma Scan } \\
\text { Garmma Scan }\end{array}$ \\
\hline Ringold & & Mtn. Whitefish & SA (April \& Oct.) & Ganma Scan \\
\hline \multicolumn{5}{|c|}{ Ducks $(b)$} \\
\hline Vicinity $100-\mathrm{N}$ & $\begin{array}{l}6451 \\
\text { ATS* }\end{array}$ & $\begin{array}{l}\text { Mallard } \\
\text { Other }\end{array}$ & $\begin{array}{l}2 / \mathrm{mo} .(\mathrm{c}) \\
2 / \mathrm{mo} .(\mathrm{c})\end{array}$ & $\begin{array}{l}\text { Gamma Scan } \\
\text { Gamma Scan }\end{array}$ \\
\hline B Pond & $\begin{array}{l}1298 \\
\text { ATS* }\end{array}$ & $\begin{array}{l}\text { Mallard } \\
\text { Other }\end{array}$ & $\begin{array}{l}2 / \mathrm{mo} .(\mathrm{c}) \\
2 / \mathrm{mo} .(\mathrm{c})\end{array}$ & $\begin{array}{l}\text { Gamma Scan } \\
\text { Gamma Scan }\end{array}$ \\
\hline Gable Pond & $\begin{array}{l}0432 \\
\text { ATS* }\end{array}$ & $\begin{array}{l}\text { Mallard } \\
\text { Other }\end{array}$ & $\begin{array}{l}2 / \mathrm{mo} .(\mathrm{c}) \\
2 / \mathrm{mo} .(\mathrm{c})\end{array}$ & $\begin{array}{l}\text { Gamma Scan } \\
\text { Gamma Scan }\end{array}$ \\
\hline U Pond & $\begin{array}{l}0439 \\
\text { ATS* }\end{array}$ & $\begin{array}{l}\text { Mallard } \\
\text { Other }\end{array}$ & $\begin{array}{l}2 / \mathrm{mo} .(\mathrm{c}) \\
2 / \mathrm{mo} .(\mathrm{c})\end{array}$ & $\begin{array}{l}\text { Gamma Scan } \\
\text { Gamma Scan }\end{array}$ \\
\hline West Lake & $\begin{array}{l}6186 \\
\text { ATS* }\end{array}$ & $\begin{array}{l}\text { Mallard } \\
\text { Other }\end{array}$ & $\begin{array}{l}2 / \mathrm{mo} .(\mathrm{c}) \\
2 / \mathrm{mo} .(\mathrm{c})\end{array}$ & $\begin{array}{l}\text { Ganma Scan } \\
\text { Gamma Scan }\end{array}$ \\
\hline 300 Area & $\begin{array}{l}1589 \\
\text { ATS* }\end{array}$ & $\begin{array}{l}\text { Mallard } \\
\text { Other }\end{array}$ & $\begin{array}{l}2 / \mathrm{mo} .(\mathrm{c}) \\
2 / \mathrm{mo} .(\mathrm{c})\end{array}$ & $\begin{array}{l}\text { Garmma Scan } \\
\text { Gamma Scan }\end{array}$ \\
\hline \multicolumn{5}{|c|}{ Geese $^{(d)}$} \\
\hline Vicinity $100-N$ & ATS* & & $1 / \mathrm{mo} \cdot(\mathrm{e})$ & Gamma Scan \\
\hline \multicolumn{5}{|c|}{ Game Birds $(f)$} \\
\hline \multicolumn{5}{|l|}{$\begin{array}{l}\text { Vicinity Burial } \\
\text { Grounds: }\end{array}$} \\
\hline $100-B C$ & ATS* & $\begin{array}{l}\text { Chukar, Pheas- } \\
\text { ant or Quail }\end{array}$ & $\begin{array}{l}\text { A ( } 3 \text { Bird Comp. } \\
\text { in October) }\end{array}$ & Gamma Scan \\
\hline $100-K$ & ATS* & $\begin{array}{l}\text { Chukar, Pheas- } \\
\text { ant or Quail }\end{array}$ & $\begin{array}{l}\text { A (3 Bird Comp. } \\
\text { in October) }\end{array}$ & Gamma Scan \\
\hline
\end{tabular}

(a) Collect up to five fish at each sampling. Two samples are to be taken from each: 1) skinless muscle fillet from one side of fish and 2) remainder of fish.

(b) Other species such as coot, teal, widgeon, etc., may be used for this sample.

(c) $2 /$ mo. in January, August, and December.

(d) Collect the same species October through December.

(e) $1 /$ mo. in October, November, and December.

(f) Composite the samples only if they are of the same species; otherwise submit as separate samples.

* ATS - according to species. 
VII. WILOLIFE (contd)

\begin{tabular}{|c|c|c|c|c|}
\hline Location & EMA\# & Species & Frequency & Analyses \\
\hline & & Game Birds (contd & (a) & \\
\hline $100-N$ & ATS* & $\begin{array}{l}\text { Chukar, Pheas- } \\
\text { ant or Quai } 1\end{array}$ & $\begin{array}{l}\text { A ( } 3 \text { Bird Comp. } \\
\text { in October) }\end{array}$ & Gamma Scan \\
\hline $100-D$ & ATS* & $\begin{array}{l}\text { Chukar, Pheas- } \\
\text { ant or Quail }\end{array}$ & $\begin{array}{l}\text { A (3 Bird Comp. } \\
\text { in October) }\end{array}$ & Gamma Scan \\
\hline $100-H$ & ATS* & $\begin{array}{l}\text { Chukar, Pheas- } \\
\text { ant or Quail }\end{array}$ & $\begin{array}{l}\text { A ( } 3 \text { Bird Comp. } \\
\text { in October) }\end{array}$ & Gamma Scan \\
\hline $100-F$ & ATS* & $\begin{array}{l}\text { Chukar, Pheas- } \\
\text { ant or Quail }\end{array}$ & $\begin{array}{l}\text { A ( } 3 \text { Bird Comp. } \\
\text { in October) }\end{array}$ & Gamma Scan \\
\hline $200 E$ & ATS* & $\begin{array}{l}\text { Chukar, Pheas- } \\
\text { ant or Quail }\end{array}$ & $\begin{array}{l}\text { A (3 Bird Comp. } \\
\text { in October) }\end{array}$ & Gamma Scan \\
\hline $200 \mathrm{~W}$ & ATS* & $\begin{array}{l}\text { Chukar, Pheas- } \\
\text { ant or Quail }\end{array}$ & $\begin{array}{l}\text { A ( } 3 \text { Bird Comp. } \\
\text { in October) }\end{array}$ & Gamma Scan \\
\hline 300 Area & ATS* & $\begin{array}{l}\text { Chukar, Pheas- } \\
\text { ant or Quail }\end{array}$ & $\begin{array}{l}\text { A ( } 3 \text { Bird Comp. } \\
\text { in October) }\end{array}$ & Gamma Scan \\
\hline \multicolumn{5}{|l|}{ OFFSITE } \\
\hline Wahluke Slope & ATS* & $\begin{array}{l}\text { Chukar, Pheas- } \\
\text { ant or Quail }\end{array}$ & $\begin{array}{l}\text { A (3 Bird Comp. } \\
\text { in October) }\end{array}$ & Gamma Scan \\
\hline \multicolumn{5}{|c|}{ Deer } \\
\hline 100 Area & $\begin{array}{l}\text { According } \\
\text { to location }\end{array}$ & Mule & $2 / y r$ & $\begin{array}{l}\text { Muscle - Gamma Scan } \\
\text { Bone - } 90 \mathrm{Sr} \\
\text { Liver - Pu }\end{array}$ \\
\hline 200 Areas & $\begin{array}{l}\text { According } \\
\text { to location }\end{array}$ & Mule & $2 / y r$ & $\begin{array}{l}\text { Muscle - Gamma Scan } \\
\text { Bone - } 90 \mathrm{Sr} \\
\text { Liver - Pu }\end{array}$ \\
\hline 300 Area & $\begin{array}{l}\text { According } \\
\text { to location }\end{array}$ & Mule & $2 / y r$ & $\begin{array}{l}\text { Muscle - Gamma Scan } \\
\text { Bone - } 90 S r \\
\text { Liver - Pu }\end{array}$ \\
\hline \multicolumn{5}{|c|}{ Rabbits } \\
\hline 100-N Area & $\begin{array}{l}\text { According } \\
\text { to location }\end{array}$ & Cottontail & $4 / y r$ & $\begin{array}{l}\text { Muscle - Gamma Scan } \\
\text { Bone - } 90 \mathrm{Sr}\end{array}$ \\
\hline 200 Areas & $\begin{array}{l}\text { According } \\
\text { to location }\end{array}$ & Cottontail & $4 / y r$ & $\begin{array}{l}\text { Muscle }- \text { Ganma Scan } \\
\text { Eone }-90 s r\end{array}$ \\
\hline 300 Area & $\begin{array}{l}\text { According } \\
\text { to location }\end{array}$ & Cottontail & $4 / y r$ & $\begin{array}{l}\text { Muscle - Gamma Scan } \\
\text { Bone }-90 \mathrm{Sr}\end{array}$ \\
\hline
\end{tabular}

(a) Composite the samples only if they are of the same species; otherwise submit as separate samples.

* ATS - According to species. 
VIII. SOIL AND VEGETATION

\begin{tabular}{|c|c|c|c|c|}
\hline Location & Soil & Vegetation & Frequency & Analyses \\
\hline Benton City & 6000 & 6046 & A & $\begin{array}{l}\text { Gamma scan, } \\
90 \mathrm{Sr}, \mathrm{U}, \mathrm{Pu}(\mathrm{a})\end{array}$ \\
\hline Yakima Barricade & 6004 & 6050 & A & $\begin{array}{l}\text { Gamma scan, } \\
90 \mathrm{Sr}, \mathrm{U}, \mathrm{Pu}(\mathrm{a})\end{array}$ \\
\hline Wahluke \#2 Air Sampling Station & 6007 & 6053 & A & $\begin{array}{l}\text { Gamma scan, } \\
90 \mathrm{Sr}, \mathrm{U}, \mathrm{Pu}(\mathrm{a})\end{array}$ \\
\hline Berg Ranch Air Sampling Station & 6008 & 6054 & A & $\begin{array}{l}\text { Gamma scan, } \\
90 \mathrm{Sr}, \mathrm{U}, \mathrm{Pu}(\mathrm{a})\end{array}$ \\
\hline Ringold & 6009 & 6055 & A & $\begin{array}{l}\text { Gamma scan, } \\
90 \mathrm{Sr}, U, \mathrm{Pu}(\mathrm{a})\end{array}$ \\
\hline $\begin{array}{l}\text { Byers Landing Air Sampling } \\
\text { Station }\end{array}$ & 6011 & 6057 & A & $\begin{array}{l}\text { Gamma scan, } \\
90 \mathrm{Sr}, U, \mathrm{Pu}(\mathrm{a})\end{array}$ \\
\hline Wye Barricade & 6016 & 6062 & A & $\begin{array}{l}\text { Gamma scan, } \\
90 \mathrm{Sr}, \mathrm{U}, \mathrm{Pu}(\mathrm{a})\end{array}$ \\
\hline Hanford Townsite (CP\#57) & 6017 & 6063 & A & $\begin{array}{l}\text { Gamma scan, } \\
90 \mathrm{Sr}, \mathrm{U}, \mathrm{Pu}(\mathrm{a})\end{array}$ \\
\hline 200 E Hill Air Sampling Station & 6022 & 6068 & A & $\begin{array}{l}\text { Gamma scan, } \\
90 \mathrm{Sr}, \mathrm{U}, \mathrm{Pu}(\mathrm{a})\end{array}$ \\
\hline Taylor Flats \#2 & 6421 & 6423 & A & $\begin{array}{l}\text { Gamma scan, } \\
90 \text { Sr, U, Pu (a) }\end{array}$ \\
\hline Prosser Barricade & 6225 & 6227 & A & $\begin{array}{l}\text { Gamma scan } \\
90 \mathrm{Sr}, \mathrm{U}, \mathrm{Pu}(\mathrm{a})\end{array}$ \\
\hline East of $200 \mathrm{~W}$ Gate (CP\#2) & 6276 & 6283 & A & $\begin{array}{l}\text { Gamma scan, } \\
90 \mathrm{Sr}, \mathrm{U}, \mathrm{Pu}(\mathrm{a})\end{array}$ \\
\hline Southeast Side FFTF Site & 6277 & 6286 & A & $\begin{array}{l}\text { Gamma scan, } \\
90 \mathrm{Sr}, \mathrm{U}, \mathrm{Pu}(\mathrm{a})\end{array}$ \\
\hline East of ALE Field Lab & 6278 & 6287 & A & $\begin{array}{l}\text { Gamma scan, } \\
90 \mathrm{sr}, \mathrm{U}, \mathrm{Pu}(\mathrm{a})\end{array}$ \\
\hline $1 / 2$ Mile Northeast FFTF Site & 6282 & 6285 & A & $\begin{array}{l}\text { Gamma scan, } \\
90 \mathrm{Sr}, \mathrm{U}, \mathrm{Pu}(\mathrm{a})\end{array}$ \\
\hline Sunnys ide & 6357 & 6363 & A & $\begin{array}{l}\text { Gamma scan, } \\
90 \mathrm{Sr}, \mathrm{U}, \mathrm{Pu}(\mathrm{a})\end{array}$ \\
\hline Sagemoor Farms & 6358 & 6364 & A & $\begin{array}{l}\text { Gamma scan, } \\
90 \mathrm{Sr}, \mathrm{U}, \mathrm{Pu}(\mathrm{a})\end{array}$ \\
\hline Taylor Flats \#1 & 6420 & 6422 & A & $\begin{array}{l}\text { Gamma scan, } \\
90 \text { sr, U, Pu }(a)\end{array}$ \\
\hline
\end{tabular}

(a) Isotopic Pu. 
VIII. SOIL AND VEGETATION (contd)

\begin{tabular}{|c|c|c|c|c|}
\hline Location & Soil & Vegetation & Frequency & Analyses \\
\hline West End Fir Road & 6360 & 6366 & A & $\begin{array}{l}\text { Gamma scan, } \\
90 \mathrm{sr}, \mathrm{U}, \mathrm{Pu}(\mathrm{a})\end{array}$ \\
\hline Harris Farm & 6361 & 6367 & A & $\begin{array}{l}\text { Gamma scan, } \\
90 \text { Sr, U, Pu (a) }\end{array}$ \\
\hline 200 ENC & 6362 & 6368 & A & $\begin{array}{l}\text { Gamma scan, } \\
90 \mathrm{Sr}, U, \mathrm{Pu}_{\mathrm{U}}(\mathrm{a})\end{array}$ \\
\hline
\end{tabular}

(a) Isotopic Pu. 
IX. EXTERNAL RADIATION MEASUREMENT

Thermoluminescent Dosimeters

\begin{tabular}{|c|c|c|c|}
\hline Location & EMA\# & Frequency & Measurement \\
\hline Coyote Rapids & 6135 & M & Immersion Dose \\
\hline Richiand Pumphouse & 1715 & M & Immersion Dose \\
\hline 200 ENC $^{(a)}$ & 1467 & M & Ambient Dose \\
\hline $200 \operatorname{ESE}^{(a)}$ & 1468 & M & Ambient Dose \\
\hline $200 \operatorname{EWC}^{(a)}$ & 1470 & M & Ambient Dose \\
\hline $200 \mathrm{EEC}^{(\mathrm{a})}$ & 1469 & M & Ambient Dose \\
\hline $200 \mathrm{WEC}^{(\mathrm{a})}$ & 1473 & M & Ambient Dose \\
\hline $\operatorname{Redox}^{(a)}$ & 1474 & M & Ambient Dose \\
\hline $200 W W C^{(a)}$ & 1471 & M & Ambient Dose \\
\hline 200 WNE $^{(a)}$ & 1472 & M & Ambient Dose \\
\hline 3705 Building $(a)$ & 1486 & M & Ambient Dose \\
\hline $\operatorname{ACRMS}(a)$ & 1698 & M & Ambient Dose \\
\hline 300 Pond (a) & 1699 & M & Ambient Dose \\
\hline 300 Southwest Gate ${ }^{(a)}$ & 6163 & M & Ambient Dose \\
\hline 300 South Gate $(a)$ & 6162 & M & Ambient Dose \\
\hline$C P \# 64^{(a)}$ & 6171 & M & Ambient Dose \\
\hline $1100 \operatorname{Area}^{(a)}$ & 6398 & M & Ambient Dose \\
\hline $100-K^{(a)}$ & 1475 & M & Ambient Dose \\
\hline $100-N$ (WPPSS) $^{(a)}$ & 1476 & M & Ambient Dose \\
\hline $100-D^{(a)}$ & 1477 & M & Ambient Dose \\
\hline 100 Area Fire Station(a) & 6164 & M & Ambient Dose \\
\hline $400 E^{(a)}$ & 1729 & M & Ambient Dose \\
\hline $400 w^{(a)}$ & 6468 & M & Ambient Dose \\
\hline $400 s^{(a)}$ & 6469 & M & Ambient Dose \\
\hline $400 N(a)$ & 6470 & M & Amb ient Dose \\
\hline FFTF North & 6177 & M & Ambient Dose \\
\hline FFTF Southeast & 6178 & M & Ambient Dose \\
\hline Prosser Barricade $(a)$ & 6176 & M & Ambient Dose \\
\hline Hanford (a) & 1480 & M & Ambient Dose \\
\hline Wye Barricade (a) & 1483 & M & Ambient Dose \\
\hline Rattlesnake Springs(a) & 1485 & M & Ambient Dose \\
\hline$E R C^{(a)}$ & 1484 & M & Ambient Dose \\
\hline Yakima Barricade $(\mathrm{a})$ & 1482 & M & Ambient Dose \\
\hline Wahluke \#2(a) & 1490 & M & Ambient Dose \\
\hline
\end{tabular}

(a) Located at Air Sampling Station. 
IX. EXTERNAL RADIATION MEASUREMENT (contd)

\begin{tabular}{|c|c|c|c|}
\hline Location & EMA\# & Frequency & Measurement \\
\hline $\operatorname{Pasco}^{(a)}$ & 1488 & $M$ & Ambient Dose \\
\hline Richland (a) & 1487 & M & Ambient Dose \\
\hline Vernita $(a)$ & 1481 & M & Ambient Dose \\
\hline Benton City (a) & 1499 & M & Ambient Dose \\
\hline Othe110 (a) & 1493 & M & Ambient Dose \\
\hline Conne 1] (a) & 1494 & M & Ambient Dose \\
\hline $\operatorname{Berg} \operatorname{Ranch}(a)$ & 1491 & M & Ambient Dose \\
\hline Wahluke Wm. (a) & 1495 & M & Ambient Dose \\
\hline Cooke Brothers $(\mathrm{a})$ & 1492 & M & Ambient Dose \\
\hline Byers Landing (a) & 1498 & M & Ambient Dose \\
\hline Pettett $(a)$ & 6353 & M & Ambient Dose \\
\hline Sagemoor Farms & 6354 & M & Ambient Dose \\
\hline Fir $\operatorname{Road}(a)$ & 6356 & M & Ambient Dose \\
\hline Walla Walla ${ }^{(a)}$ & 1695 & M & Ambient Dose \\
\hline Sunnyside ${ }^{(a)}$ & 1500 & M & Ambient Dose \\
\hline $\operatorname{McNary}(a)$ & 1696 & M & Ambient Dose \\
\hline Moses Lake ${ }^{(a)}$ & 1693 & M & Ambient Dose \\
\hline Washtucna $(a)$ & 1694 & M & Ambient Dose \\
\hline Up River 100-8 Area & 6471 & M & Ambient Dose \\
\hline Below 100-B Retention Basin & 6472 & M & Ambient Dose \\
\hline Above $100-K$ Boat Ramp & 6260 & M & Ambient Dose \\
\hline Below 100-K Retention Basins & 6473 & M & Ambient Dose \\
\hline 100-N Trench Springs & 6390 & M & Ambient Dose \\
\hline Down River 100-N Trench Springs & 6474 & M & Ambient Dose \\
\hline Down River 100-D & 6475 & M & Ambient Dose \\
\hline Down River Opposite 100-D & 6261 & M & Ambient Dose \\
\hline Lower End Locke Island & 6262 & M & Ambient Dose \\
\hline White Bluffs Ferry Lanaing & 6263 & M & Ambient Dose \\
\hline White Bluffs Slough & 6476 & M & Ambient Dose \\
\hline 100-F Area Floodplain & 6477 & M & Ambient Dose \\
\hline Below 100-F & 6264 & M & Ambient Dose \\
\hline Hanford Peninsula & 6478 & M & Ambient Dose \\
\hline Hanford Powerline Crossing & 6389 & M & Ambient Dose \\
\hline Hanford Ferry Landing & 6265 & M & Ambient Dose \\
\hline
\end{tabular}

(a) Located at Air Sampling Station. 
IX. EXTERNAL RADIATION MEASUREMENT (contd)

\begin{tabular}{|c|c|c|c|}
\hline Location & EMA\# & Frequency & Measurement \\
\hline Hanford RR Track & 6266 & $M$ & Ambient Dose \\
\hline Savage Island Slough & 6479 & $M$ & Amb ient Dose \\
\hline Ringold Island & 6267 & M & Ambient Dose \\
\hline Powerline Crossing & 6268 & $M$ & Ambient Dose \\
\hline North End Wooded Island & 6480 & M & Ambient Dose \\
\hline South End Wooded Island & 6269 & $M$ & Ambient Dose \\
\hline Is land River Mile 344 & 6481 & $M$ & Ambient Dose \\
\hline Island River Mile 333 & 6482 & $M$ & Ambient Dose \\
\hline
\end{tabular}


$X$. PORTABLE INSTRUMENT SURVEYS

Columbia River Shoreline

\begin{tabular}{|c|c|c|c|}
\hline Location & $\begin{array}{l}\text { River } \\
\text { Mile }\end{array}$ & EMA\# & Instrument \\
\hline Above 100-K Boat Ramp & $382.5 P$ & 6132 & LLM, GM \\
\hline 100-N Trench Springs & $379.4 P$ & 6129 & LLM, GM \\
\hline Down River Opposite 100-0 & $376.8 \mathrm{~F}$ & 6241 & LLM, GM \\
\hline Lower End Locke Island & 371.0 IP & 6123 & LLM, GM \\
\hline White Bluffs Ferry Landing & $369.7 P$ & 6121 & LLM, GM \\
\hline Below 100-F & $368.3 P$ & 6120 & LLM, GM \\
\hline Hanford Powerl ine Crossing & $362.6 \mathrm{P}$ & 6118 & LLM, GM \\
\hline Hanford Ferry Landing & $362.0 \mathrm{~F}$ & 6117 & LLM, GM \\
\hline Hanford Railroad Track & $361.4 P$ & 6242 & LLM, GM \\
\hline Ringold Is land & 354.7 IP & 6114 & LLM, GM \\
\hline Powerline Crossing & $350.4 P$ & 6113 & LLM, GM \\
\hline
\end{tabular}

Roadway Survey

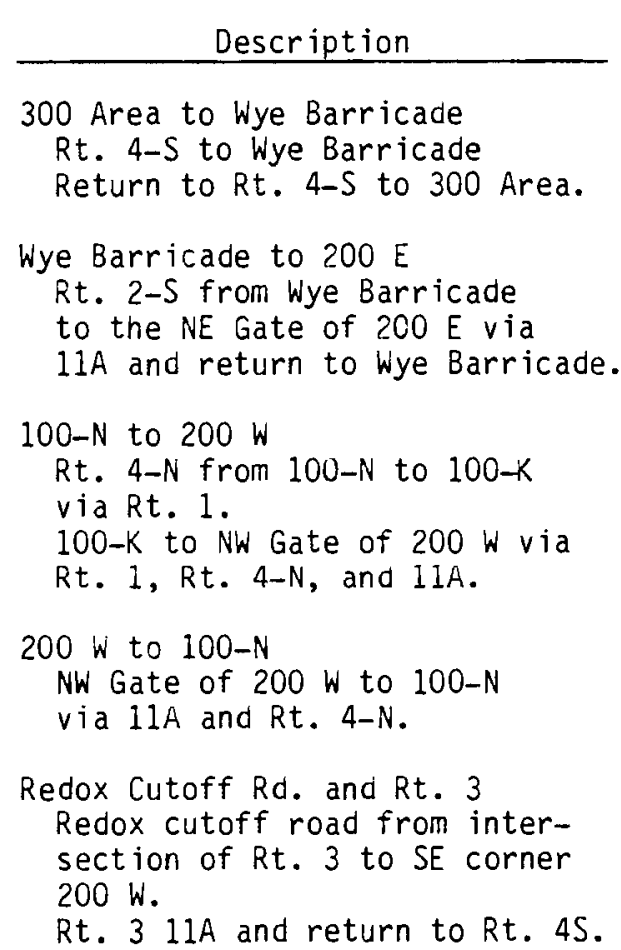

\begin{tabular}{|c|c|c|}
\hline $\begin{array}{c}\text { Indent if ication } \\
\text { Number } \\
\end{array}$ & Frequency & Instrument \\
\hline 1 & $M$ & $\begin{array}{c}\text { Bioplastic } \\
\text { Crystal }\end{array}$ \\
\hline 2 & $M$ & $\begin{array}{c}\text { Bioplastic } \\
\text { Crystal }\end{array}$ \\
\hline 3 & M & $\begin{array}{c}\text { Bioplastic } \\
\text { Crystal }\end{array}$ \\
\hline 4 & M & $\begin{array}{c}\text { Bioplastic } \\
\text { Crystal }\end{array}$ \\
\hline 5 & $M$ & $\begin{array}{c}\text { Bioplastic } \\
\text { Crystal }\end{array}$ \\
\hline
\end{tabular}


$X$. PORTABLE INSTRUMENT SURVEYS (contd)

Roadway Survey (contd)

Description

Hanford to 100-N

Rt. 2-N from Rt. $11 \mathrm{~A}$ to $100-\mathrm{N}$ intersection via Rt. 4-N.

100-N intersection to Rt. $11 \mathrm{~A}$

via Rt. $4-N$ and Rt. $2-N$.

$11 A$ to $100-K$

$11 A$ and Rt. 6 intersection to

$100-K$ via Rt. 6 and Rt. 1.

Return via Rt. 1 and Rt. 6

to $11 A$.

Yakima Barricade to NECO Entrance

Via $11 A$ and Rt. 4-S

Horn Rapids to $11 \mathrm{~A}$

Rt. 10 from Horn Rapids to

Wye Barricade.

Rt. 4-S from wye Barricade to $11 A$.

Railway Survey

300 Area to May Junction

including FFTF Spur

May to Ethel

100-N to Nancy to Helen

Helen via Audrey to $100-K$, Helen

to Susie, Susie to 200 West,

Susie to 200 East

100-K to Audrey

Audrey to 100-B, Audrey to

Vernita Highway

May to Nancy via Ruth and Bettie

Aerial Survey

Project Perimeter
Indentification

Number

Frequency

Q

Instrument

6

Bioplastic

Crystal

7

Q

Bioplastic Crystal

8

Q

Bioplastic Crystal

9

M

Bioplastic Crystal

1

2

3

Q

Bioplastic Crystal

Bioplastic Crystal

Bioplastic Crystal

4

A

Bioplastic Crystal

5

A

Bioplastic Crystal

3
A

NaI Crystal 
XI. SURVEILLANCE OF WASTE DISPOSAL SITES

Active, inactive, and retired waste disposal sites require periodic monitoring to assure appropriate maintenance. The following sites are routinely checked for vegetation growth, evidence of burrowing animals, erosion, status of enclosure, etc.

\begin{tabular}{|c|c|}
\hline Description & Frequency \\
\hline 100-K Trench & A \\
\hline 100-BC SE B. G. (105-C Solid waste) & SA \\
\hline 100-BC SW B. G. (105-B Solid waste, N. Solid waste) & SA \\
\hline 100-BC Construction B. G. & SA \\
\hline 100-BC B. G. East of 108-B & SA \\
\hline 100-BC Irradiated Metal Storage Basin Waste & SA \\
\hline 108-B Ball 3x Burial Ground & SA \\
\hline $108-B$ Crib & SA \\
\hline 105-C Trench & SA \\
\hline 105-B Trench & SA \\
\hline 107 Basin Sludge Burial & SA \\
\hline 105-C Metal Examination Waste Tank & SA \\
\hline 100-BC Overflow Pluto Crib & SA \\
\hline 107-C Retention Basin & SA \\
\hline 107-B Retention Basin & SA \\
\hline 100-BC Effluent Diversion Box & SA \\
\hline 100-BC Minor B. G.'s East of 105-B & SA \\
\hline 100-BC Outfall Structures & SA \\
\hline 100-DR Outfall Structures & SA \\
\hline 100-DDR Trench & SA \\
\hline 107-D Retention Basin & SA \\
\hline 107-DR Retention Basin & SA \\
\hline 100-DDR Effluent Lines & SA \\
\hline 100-D Dummy Decontamination Waste & SA \\
\hline 100-DDR Solid Waste B. G. (VSR Thimbles) & SA \\
\hline 100-DDR Construction B. G. & $S A$ \\
\hline $100-D D R \# 3$ B. G. NE of DR & SA \\
\hline 100-DDR Pluto Crib & SA \\
\hline 100-DDR 105 Basin Sludge B. G. & SA \\
\hline 100-ODR \#1 B. G. & SA \\
\hline 100-DDR \#2 B. G. & SA \\
\hline 100-H Trench & SA \\
\hline 107-H Basin & SA \\
\hline 100-H Effluent Lines (Junction Boxes) & SA \\
\hline
\end{tabular}


XI. SURVEILLANCE OF WASTE DISPOSAL SITES (contd)

100-H Liquid Waste Burial

$100-H \# 1$ B. G.

$S A$

$100-H$ \#2 B. G.

$S A$

P-11 Area

A

100-F Lewis Canal

$S A$

100-F Swampy Area SA

100-F Trench SA

100-F Retention Basin SA

100-F Trench Drain and Adjacent Wood Covered Pit SA

100-F Ball Washer Crib SA

$100 \mathrm{~F} \# 3 \mathrm{~B}$. G.

$100-F$ \#2 B. G. $S A$

$100-F$ \#1 B. G. SA

100-F Sawdust Burial SA

100-F Leaching Trench SA

100-F 60" Overground Pipe SA

100-F Happy Valley Farm Plots SA

200 W New Redox Pond (216-S-16) SA

200 W 01d Redox Pond (216-S-17) A

$200 \mathrm{~W} U$ Pond Overflow (216-U-11) SA

200 E B Pond (216-B-3) Q

200 E B Pond Ditch \#3 SA

200 E Purex Crib \#1 (216-A-6) SA

200 E Purex Crib \#2 (216-A-30-1) SA

200 E North of Purex Crib \#3 (216-A-37-1) SA

200 E NE Perimeter Fence SA

200 E 216-BC Crib Area SA

200 E California Nuclear B. G. Perimeter Q

300 Area $300 \mathrm{~N}$ B. G. SA

300 Area 300 wye B. G. SA

300 Area \#2 B. G. SA

300 Area \#3 B. G. SA

307 Area \#4 B. G. SA

300 Area \#5 B. G. SA

300 Area \#7 B. G SA

300 west B. G. SA

300 Area N. Process Trench at Perimeter Fence Q

$200-N, P$, and $R$ Areas SA 
,

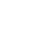


PNL -3639

UC-41

DISTRIBUTION

No. of

Copies

OFFSITE

A. A. Churm DOE Patent Group Department of Energy 9800 South Cass Ave.

Argonne, IL 60439

27 DOE Technical Information Center

R. R. Mooney Washington State Department of Social and Health Services 1514 Smith Tower

Seattle, WA 98104

ONSITE

9 DOE Richland Operations Office

0. J. Elgert

D. R. Elle (5)

H. E. Ransom

M. W. Tiernam

R. E. Austin

7 Rockwell Hanford Company

R. D. Fox

W. F. Heine

P. G. Lorenzini

W. H. Price

D. L. Uhl

R. E. Wheeler

Rockwell Files
No. of

Copies

Hanford Environmental Health Foundation

B. D. Reinert

3 UNC Nuclear Industries, Inc.

J. J. Dorian

E. A. Week ly

UNI Files

2 Westinghouse Hanford Company

P. R. Prevo

R. B. Hall

48 Pacific Northwest Laboratory

L. L. Belt

P. J. Blumer (20)

P. E. Bramson

C. S. Cline

J. P. Corley

R. L. Dirkes

P. A. Eddy

L. W. Hanke 1

J. D. Harrison

W. W. King

H. V. Larson

W. D. McCormack

V. L. McGhan

D. L. Merrill

K. R. Price

M. R. Quarders (3)

B. D. Robertson

M. J. Sula

J. S. Wilbur

R. H. Williams

Technical Information (5)

Publishing Coordination GO (2) 
